\title{
EVALUACIÓN DE LA FRAGILIDAD DE DOS SOLUCIONES DE REHABILITACIÓN PARA UN EDIFICIO CON PLANTA BAJA DÉBIL DAÑADO DURANTE EL SISMO 19/S17
}

\author{
Sonia E. Ruiz Gómez ${ }^{(1)}$, René Jiménez Jordán ${ }^{(2)}$, Marco A. Santos Santiago ${ }^{(2)}$ y \\ Miguel A. Orellana Ojeda ${ }^{(2)}$
}

\begin{abstract}
RESUMEN
La fragilidad sísmica de un edificio rehabilitado con diferentes técnicas puede ser muy diferente. Aquí se demuestra que dicha fragilidad puede ser muy similar cuando un edificio con planta baja débil (PBD) se rehabilita mediante encamisado de concreto y, alternativamente, usando contravientos restringidos al pandeo (CRP). Para ello se analiza un edificio de 5 niveles con PBD, localizado en un sitio en la Ciudad de México (CDMX) donde se presentaron daños estructurales durante el sismo del 19 de septiembre de 2017 (19/S17). El edificio está formado en su PB por marcos de concreto reforzado y por mampostería confinada y reforzada con acero horizontal en sus pisos superiores. Se analizan tres casos: S1) que corresponde a la estructura original con PBD; S2) en la que se encamisan con concreto las columnas de la PB; y S3) en la que las columnas de la PB se cubren con ángulos y soleras de acero y, se añaden CRP; en los casos S2 y S3 se rehabilitan los muros con malla electrosoldada y aplanado. Para el análisis se utilizan diez acelerogramas registrados en la CDMX, durante el sismo 19/S17, en suelo con periodo dominante cercano a 1.0s. Se presentan los perfiles de distorsiones máximas de entrepiso (DME) correspondientes a los tres casos, sujetos a la acción de los diez movimientos con diferentes intensidades, lo que es útil para entender el comportamiento de los sistemas estructurales. Se obtienen curvas de fragilidad para los valores límite de DME recomendados por las Normas Técnicas Complementarias para Diseño por Sismo (2017).
\end{abstract}

Palabras clave: curvas de fragilidad; piso bajo blando; edificio rehabilitado; disipadores de energía; contravientos restringidos al pandeo

\footnotetext{
Artículo recibido el 21 de julio de 2018 y aprobado para su publicación el 11 de marzo de 2020. Se aceptarán comentarios y/o discusiones hasta cinco meses después de su publicación.

(1) Instituto de Ingeniería, Universidad Nacional Autónoma de México, Circuito Interior s/n, Ciudad Universitaria, Delegación Coyoacán, México, C.P. 0.4510, Tel. (55)5623-3654. SRuizG@,iingen.unam.mx

(2) Instituto de Ingeniería, Universidad Nacional Autónoma de México, Circuito Interior s/n, Ciudad Universitaria, Delegación Coyoacán, México, C.P. 0.4510, Tel. (55)5623-3600. RJimenezJ@iingen.unam.mx, MSantosS@iingen.unam.mx, MOrellanaO@iingen.unam.mx
}

DOI: $10.18867 /$ ris. 102.513 


\title{
FRAGILITY ASSESSMENT OF TWO REHABILITATION SOLUTIONS FOR A FIRST SOFT STORY BUILDING DAMAGED DURING THE 19/S17 MEXICO EARTHQUAKE
}

\begin{abstract}
The seismic fragility of a building rehabilitated with different techniques can be very different. Here it is shown that such fragility can be similar when a soft-first story (SFS) building is rehabilitated though concrete jacketing, and alternatively, using buckling restrained braces (BRB). For this aim, it is analyzed a 5-story building with soft first story, located in a site of Mexico City where several structures were damaged during the September 17, 2017 (19/S17) earthquake. The building is constituted by regular R/C moment-resisting frames in the soft story, and confined masonry walls with horizontal steel bars in the upper levels. Three cases are analyzed: S1) which correspond to the original structure with SFS; S2) in which concrete jacketing is used in the columns of the first soft story; and S3) in which steel jacketing and buckling-restrained braces (BRB) are added to the first story; in the cases S2 and S3 the masonry walls are retrofitted with steel mesh and mortar. Ten seismic ground motions are used to excite the structures; they correspond to accelerograms recorded in Mexico City, during the 19/S17 seismic event, in soil with dominant period around 1.0s. Story drifts of the three buildings corresponding to the ten seismic ground motions with different intensities, are presented. Fragility curves of the buildings are calculated for values of the maximum story drifts recommended by the Mexico City Building Code (2017).
\end{abstract}

Keywords: vulnerability assessment; soft first story; rehabilitation of buildings; energy dissipating devices; buckling restrained braces

\section{INTRODUCCIÓN}

La construcción de edificios con planta baja débil es muy común en mega-ciudades y en zonas urbanas con espacio limitado. Esta situación no es la excepción en la Ciudad de México en donde es común construir edificios cuyo piso bajo se utiliza como estacionamiento, lobby o área comercial.

Durante el sismo del 19 de septiembre de 2017 (19/S17) el porcentaje de colapsos correspondientes a piso bajo débil (PBD) fue $35.3 \%$ (dato obtenido de un estudio hecho por los autores) Este alto porcentaje llama la atención y conduce a la necesidad de estudiar el comportamiento y fragilidad de edificios con este tipo de estructuración, así como de revisar la seguridad de los edificios de este tipo que, aunque no fallaron durante el evento, es necesario evaluar y, en su caso, rehabilitar con el fin de que cuenten con suficiente confiabilidad estructural y se garantice su buen comportamiento en futuros eventos sísmicos.

Los cerca de 14 edificios con PBD que colapsaron durante el sismo 19/S17 se encontraban, en general, desplantados en suelos con periodos dominantes cercanos a $1 \mathrm{~s}$. De los edificios colapsados, $41.7 \%$ correspondieron a edificios de 5 niveles, $25 \%$ a edificios de 6 niveles, $16.7 \%$ a 3 niveles, y el $8.3 \%$ de 7 y 8 niveles. Es decir, la mayoría de los edificios con PBD colapsados fueron de mediana altura con 5 o 6 niveles. Esta información se obtuvo a partir de un estudio breve realizado por los autores. El hecho de que colapsaran edificios con 5 o 6 niveles es debido en gran parte a que dichos edificios generalmente presentan periodos fundamentales de vibración menores a 1s, pero al sufrir "ablandamiento estructural" su periodo se incrementa, acercándose al periodo "pico" del espectro de aceleraciones, presentando una especie de "resonancia" estructural; además, en la zona de la CDMX con periodo dominante cercano a 1s, es más 
frecuente encontrar edificios de mediana altura con 5 - 6 niveles, que edificios con mayor número de pisos.

En la literatura existen diversos estudios que tratan sobre la fragilidad de este tipo de estructuración y su posible mecanismo de falla de tipo frágil (Chopra et al., 1973; Miyamoto y Scholl, 1996; Beigi et al., 2014; Choudhry y Kaushik, 2018). Por otro lado, en México se han realizado estudios sobre edificios con PBD en donde se considera la influencia de movimientos sísmicos que son de banda angosta, como los que ocurren en la zona de terreno blando de la Ciudad de México (Ruiz et al., 1989; Esteva, 1992; Tena Colunga, 2010; Hernández García y Tena Colunga, 2016).

Las referencias antes mencionadas presentan contribuciones importantes que hacen entender el fenómeno, sensibilizarse sobre las variables que son significativas en el comportamiento estructural, y dar posibles soluciones de diseño estructural; sin embargo, ninguna de ellas muestra que usando una técnica de rehabilitación a base de dispositivos disipadores de energía histeréticos más encamisado de columnas con acero, es posible obtener un nivel de confiabilidad similar a la que proporciona una técnica de rehabilitación tradicional (encamisado de columnas con concreto).

En el presente estudio se demuestra lo anterior mediante la evaluación de la fragilidad de un edificio de 5 niveles con PBD rehabilitado con las siguientes dos técnicas: 1) encamisado de concreto reforzado de las columnas de la planta baja (PB), y 2) inclusión de contravientos restringidos al pandeo (CRP) más encamisado de columnas con ángulos y soleras de acero, en la PB del edificio; en ambos casos fue necesario rehabilitar los muros de los niveles superiores mediante malla electrosoldada y aplanado. Los CRP han sido ampliamente estudiado en México debido a las ventajas que presentan para edificios tanto nuevos como existentes (Terán y Virto-Cambray, 2009; Guerrero et al., 2016a, 2016b).

Aunque la mayoría de los edificios colapsados en el sismo de 19/S17 fueron diseñados y construidos previamente al sismo de 1985, el presente trabajo se enfoca al estudio un edificio diseñado posterior a la publicación del Reglamento de Construcciones para el Distrito Federal (RCDF) 2004 (lo que obedece, en parte, al gran crecimiento en la construcción de vivienda en los años recientes). El edificio en estudio se cataloga como de "piso bajo débil" debido a que se supone que en el diseño no se siguieron en forma estricta las recomendaciones del RCDF 2017, por lo que necesita ser rehabilitado.

\section{CASOS DE ESTUDIO}

El edificio por estudiar consta de 5 niveles, el cual se rehabilita con dos técnicas diferentes. La estructura presenta planta rectangular, e irregularidad en su altura, caracterizada por un cambio de rigidez de entrepiso de la planta baja (PB) al segundo entrepiso. Las figuras 1 y 2 muestran la planta (PB y nivel 1), y la elevación del edificio, respectivamente.

En el presente estudio se supone que el edificio original fue diseñado después del año 2004 siguiendo las especificaciones de las Normas Técnicas Complementarias para Diseño por Sismo (NTC-S2004), para Diseño y Construcción de Estructuras de Concreto (NTC-C2004), y de Mampostería (NTC-M2004). El edificio se ubica entre las fronteras de la zona de transición y zona de terreno blando tipo IIIa. De acuerdo con las NTC-S2004 se supone que el edificio debe ser diseñado para la zona que proporcione el espectro de mayores aceleraciones, por lo que aquí se elige el correspondiente a la zona IIIa. 


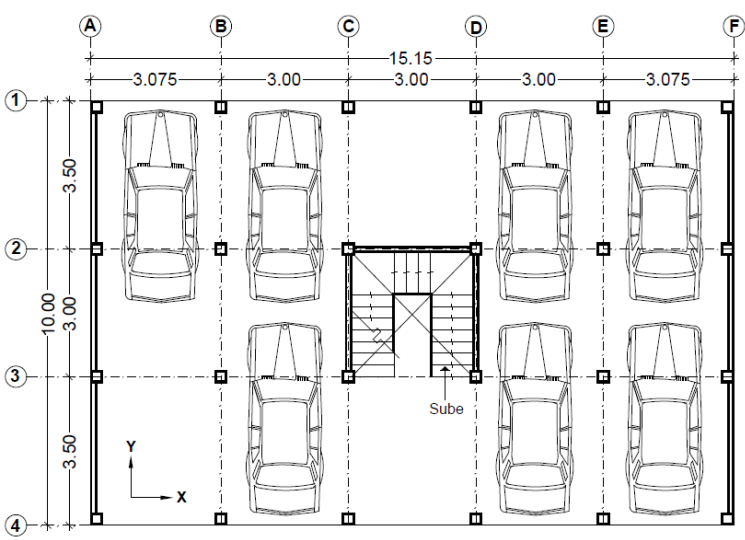

a) Planta baja (PB)

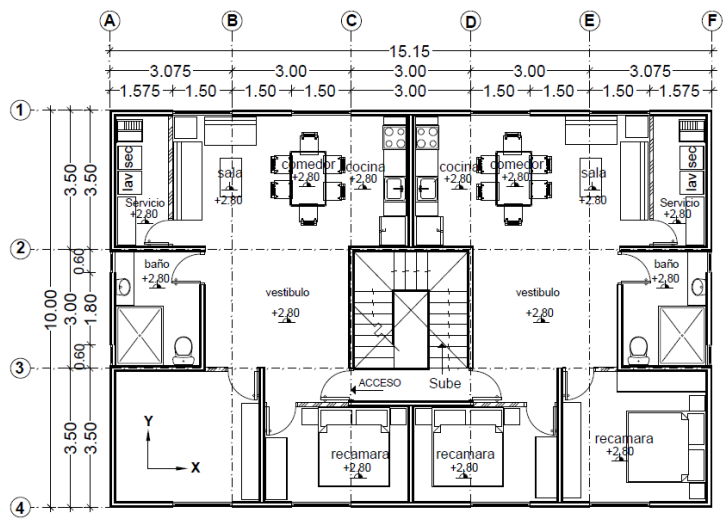

b) Nivel 1

Figura 1. Distribución de elementos estructurales en planta

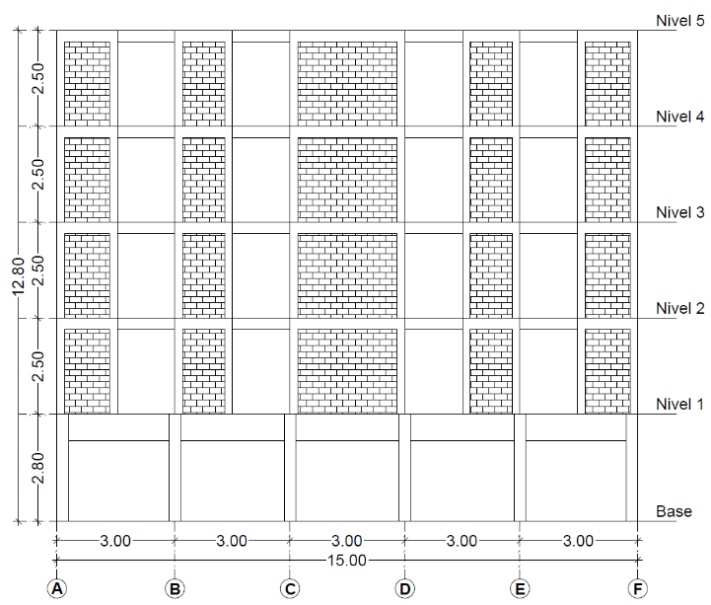

Figura 2. Distribución de elementos estructurales en altura

El sistema estructural original está formado por marcos de concreto reforzado en planta baja, y por muros de mampostería confinada de piezas macizas y reforzada con acero horizontal del nivel 1 al nivel azotea. Se supuso que la capacidad resistente entre fuerza cortante es menor que 60 por ciento de la misma relación correspondiente al segundo entrepiso, por lo que el edificio se clasifica como "edificio con piso bajo débil" de acuerdo con las NTC-S2017, y como consecuencia requiere ser rehabilitado. Se hace la observación de que si se hubieran respetado las NTC-C2004, el edificio no requeriría ser rehabilitado (Cabrera y Ruiz, 2019).

El espectro de diseño empleado para el diseño se muestra en la figura 3, el cual corresponde a terreno tipo IIIa, según el cuerpo principal de las NTC-S2004.

Los marcos de concreto se modelaron usando elementos barra, y los muros de mampostería se modelaron con el método de la columna ancha (Pérez-Gavilán et al., 2015) suponiendo falla por fuerza cortante. Se supone un factor de comportamiento sísmico $Q=2$. Los efectos de irregularidad se consideraron de acuerdo con la sección 6 de las NTC-S2004; se trata de una estructura fuertemente irregular. Las 
secciones de los elementos estructurales del edificio original (S1) se muestran en la tabla 1.

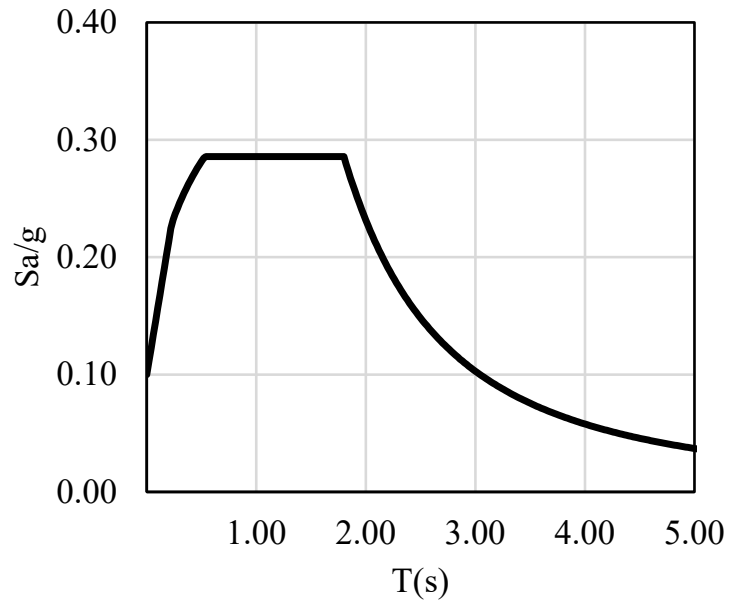

Figura 3. Espectros de diseño sísmico utilizado para el diseño del edificio original

Tabla 1. Dimensiones de los elementos estructurales

\begin{tabular}{ccc}
\hline Elemento & Dimensiones $(\mathrm{m})$ & Nivel \\
\hline Columnas PB & $0.30 \times 0.30$ & PB-N1 \\
Vigas N1 & $0.30 \times 0.70$ & N1 \\
Vigas niveles superiores & $0.12 \times 0.30$ & N2-N5 \\
Muro de mampostería & $0.12 \times 1.50$ & N2-N5 (Dirección X) \\
Muro de mampostería & $0.12 \times 1.80$ & N2-N5 (Dirección Y) \\
Muro de mampostería & $0.12 \times 2.10$ & N2-N5 (Dirección Y) \\
Muro de mampostería & $0.12 \times 2.25$ & N2-N5 (Dirección X) \\
Muro de mampostería & $0.12 \times 2.60$ & N2-N5 (Dirección Y) \\
Muro de mampostería & $0.12 \times 2.70$ & PB-N1 (Dirección Y) \\
Muro de mampostería & $0.12 \times 3.00$ & N2-N5 (Dirección X y Y) \\
Muro de mampostería & $0.12 \times 3.20$ & PB-N1 (Dirección Y) \\
Muro de mampostería & $0.12 \times 3.50$ & N2-N5 (Dirección Y) \\
\hline
\end{tabular}

En lo que sigue se describen las características generales de los tres casos de interés (edificio original (S1), y dos edificios rehabilitados (S2 y S3)):

1. Caso S1. Corresponde al edificio original diseñado de acuerdo con las NTC-S2004. Dado que el diseño no respetó todas las especificaciones del RCDF 2004, el edificio se clasifica como edificio con planta baja débil de acuerdo con las condiciones que se especifican en las NTC-S2017.

2. Caso S2. Corresponde al caso S1 después de ser rehabilitado mediante la técnica de encamisado de columnas y añadiendo malla electrosoldada y aplanado a los muros de los niveles superiores. E1 diseño se realizó de acuerdo con las NTC-S2017. En el diseño rigió el requerimiento de "columna fuerte - viga débil". El edificio rehabilitado no se cataloga como de planta baja débil, cuando se aplica la definición de las NTC-S2017. El espectro que se empleó para el diseño de la rehabilitación es el que se presenta en la figura 4 (línea punteada), el cual corresponde a los siguientes parámetros: factor de comportamiento sísmico $\mathrm{Q}=2$, factor de sobre-resistencia $\mathrm{R}_{0}=2$, factores de hiperestaticidad $\mathrm{k}_{1}=1$ y $\mathrm{k}_{2}=0.2$, y factor de irregularidad de 0.8. En la tabla 2 se muestran las secciones de las columnas y vigas correspondientes a la planta baja del edificio. 


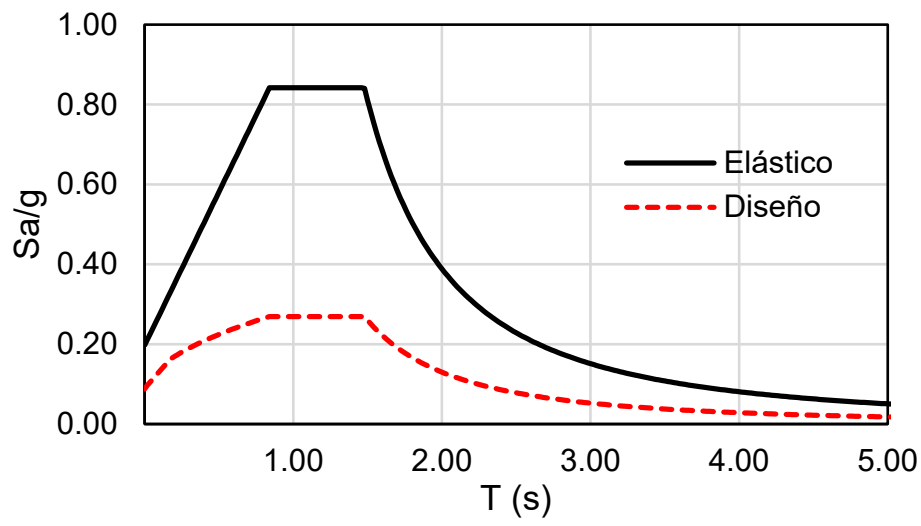

\begin{tabular}{cc}
\hline $\mathrm{T}_{\mathrm{s}}$ & 0.793 \\
$\mathrm{a}_{0}$ & 0.197 \\
$\mathrm{c}$ & 0.842 \\
$\mathrm{~T}_{\mathrm{a}}$ & 0.84 \\
$\mathrm{~T}_{\mathrm{b}}$ & 1.479 \\
$\mathrm{k}$ & 0.655 \\
\hline
\end{tabular}

Figura 4. Espectro de diseño y sus correspondientes parámetros

Tabla 2. Dimensiones de columnas y vigas de la planta baja. Caso S2

\begin{tabular}{ccc}
\hline Elemento & $\begin{array}{c}\text { Dimensiones } \\
\text { de columnas } \\
(\mathrm{m})\end{array}$ & Nivel \\
\hline Columnas PB & $0.45 \times 0.45$ & PB-N1 \\
Vigas PB & $0.30 \times 0.70$ & $\mathrm{~N} 1$ \\
\hline
\end{tabular}

3. Caso S3. Corresponde al caso S1 después de añadir contravientos de pandeo restringido, encamisar las columnas (utilizando ángulos y soleras de acero) en su planta baja, y añadir malla electrosoldada y aplanado a los muros de los niveles superiores. El diseño del refuerzo se realizó de tal forma que tanto la rigidez como la resistencia lateral fuesen similares a las del edificio correspondiente al caso S2. Las columnas de la PB se encamisaron con soleras de 3/16" de espesor y 2" de peralte, de acero estructural ASTM-A-36 con esfuerzo de fluencia $f_{y}=2,530 \mathrm{~kg} / \mathrm{cm}^{2}$. La ubicación y orientación de los CRP se indican con línea punteada en las figuras 5 a y 5 b. Sus dimensiones se muestran en la tabla 3. El espectro de diseño que se utilizó para la rehabilitación es similar al de la figura 6 , con la diferencia que para este caso se usó un factor de irregularidad igual a 0.7 en vez de 0.8 . Se hace la observación que los CRP del caso 3 no se diseñaron siguiendo las recomendaciones de las NTCS2017 (en donde se permite utilizar un factor de comportamiento sísmico $Q=4$ ), sino que la estructura se diseñó de manera que tanto la rigidez como la resistencia lateral del sistema fuesen similares a las de la estructura del caso 2. Esto se hizo con el fin de demostrar que, si se rehabilita el edificio con CRP, la fragilidad estructural puede ser similar a la de la estructura rehabilitada con un método tradicional, que es el objetivo del presente estudio. En el caso de que los CRP se hubiesen diseñado con las NTC-S2017 (suponiendo Q = 4), las dimensiones de los contravientos habrían sido ligeramente menores (Santos et al., 2017; Ruiz, 2019), dando lugar a una fragilidad mayor a la correspondiente al edificio del caso S2 (Jiménez Jordán, 2018; Ruiz et al., 2019).

Tabla 3. Dimensiones de CPR. Caso CS3

\begin{tabular}{ccc}
\hline Elemento & $\begin{array}{c}\text { Área del } \\
\text { núcleo }\left(\mathbf{m}^{\mathbf{2}}\right)\end{array}$ & Longitud (m) \\
\hline CPR-1 & 0.0018 & 3.36 \\
\hline
\end{tabular}




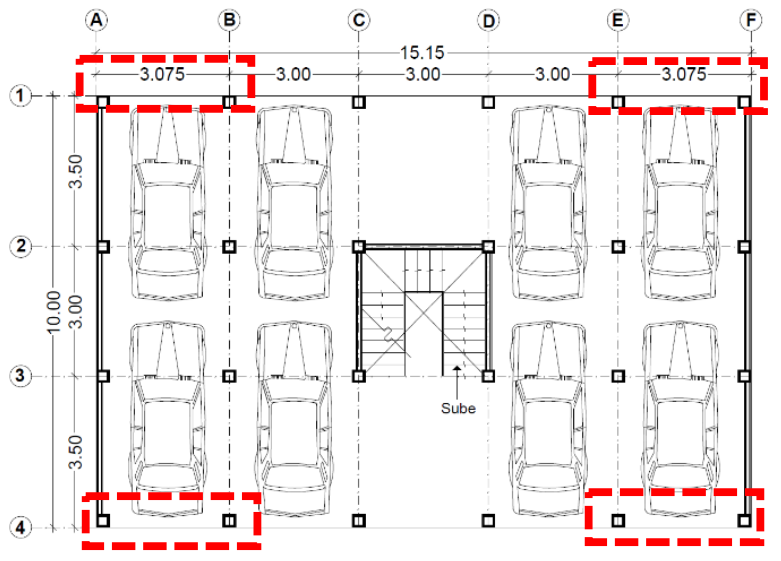

a) Ubicación de los CPR en planta

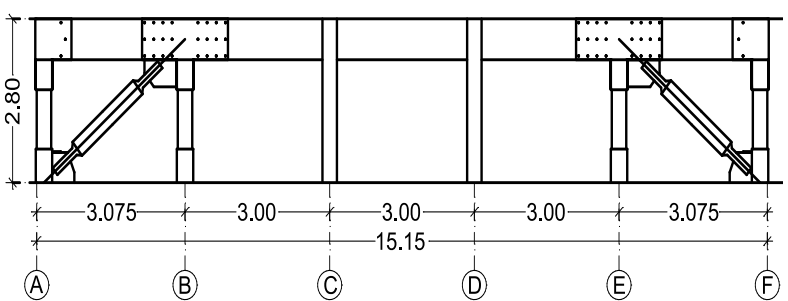

b) Configuración de la planta baja en elevación

Figura 5. Ubicación de los contravientos de pandeo restringido. Caso S3

Los periodos fundamentales de vibración de los casos en estudio se muestran en la tabla 4. El detalle del diseño y armado de los elementos de los casos S1, S2 y S3 se puede encontrar en Jiménez Jordán (2018).

Tabla 4. Periodos de vibración

\begin{tabular}{cc}
\hline Caso & Periodo (s) \\
\hline S1 & 0.42 \\
S2 & 0.31 \\
S3 & 0.32 \\
\hline
\end{tabular}

\section{SELECCIÓN Y ESCALAMIENTO DE MOVIMIENTOS SÍSMICOS}

Con el fin de realizar el análisis de fragilidad de los casos S1, S2 y S3 se seleccionaron diez acelerogramas correspondientes a movimientos del suelo registrados durante el sismo 19/S17 en terreno de transición de la Ciudad de México, con un periodo dominante cercano a $1.0 \mathrm{~s}$. En la tabla 5 se listan los datos de las estaciones que registraron el movimiento, la institución que lo realizó (CIRES), la ubicación de la estación (latitud y longitud), y la aceleración máxima del suelo ( $\left.\mathrm{A}_{\text {máx }}\right)$ como fracción de la gravedad $(\mathrm{g})$. Se hace la observación que en la tabla 5 no se listan otros registros correspondientes a movimientos ocurridos durante el evento 19/S17 y registrados en la zona de transición (por ejemplo, no aparece la componente S00E de los registros en las estaciones JC54 y CH84), debido a que cuando se realizó el presente estudio existía la duda sobre la precisión de estos registros sísmicos y las causas del porqué presentaban variaciones importantes con respecto a su correspondiente componente ortogonal. En la figura 6a se muestran los correspondientes espectros elásticos de pseudo-aceleración de los registros sísmicos correspondientes a la tabla 5 , para un porcentaje de amortiguamiento crítico $\zeta=5 \%$.

La duración de los movimientos se recortó tal que correspondiera a un intervalo del 5\% al 95\% de la intensidad de Arias. Se filtraron las frecuencias menores que $0.1 \mathrm{~Hz}$, y mayores que $10 \mathrm{~Hz}$. Las señales se escalaron con la medida de intensidad basada en el promedio de las ordenadas espectrales $S a_{\text {prom }}$ (Baker y Cornell, 2006), de acuerdo con la ecuación 1. 
Tabla 5. Características de los movimientos sísmicos

\begin{tabular}{cccccc}
\hline Movimiento & Estación & Componente & Latitud & Longitud & $\mathrm{A}_{\operatorname{máx}}(\mathrm{g})$ \\
\hline 1 & ES57 & S00E & 19.4017 & 99.1775 & 0.066 \\
2 & ES57 & N90W & 19.4017 & 99.1775 & 0.073 \\
3 & SI53 & S00E & 19.3753 & 99.1483 & 0.133 \\
4 & SI53 & N90W & 19.3753 & 99.1483 & 0.182 \\
5 & JC54 & N00E & 19.3130 & 99.1272 & 0.269 \\
6 & CH84 & N00E & 19.3300 & 99.1254 & 0.144 \\
7 & LEAC & N00E & 19.3227 & 99.0976 & 0.155 \\
8 & LEAC & N90W & 19.3227 & 99.0976 & 0.155 \\
9 & AE02 & N00E & 19.4290 & 99.0584 & 0.096 \\
10 & AE02 & N90W & 19.4290 & 99.0584 & 0.109 \\
\hline
\end{tabular}

$\operatorname{Sa} a_{\text {prom }}\left(T_{1}, \ldots, T_{n}\right)=\left(\prod_{i=1}^{n} S a\left(T_{i}\right)\right)^{1 / n}$

donde $T_{1}, \ldots, T_{n}$ son los $n$ periodos en el intervalo de interés. En el presente trabajo se considera que con incrementos de periodo de $0.01 \mathrm{~s}$ se captura con buena aproximación la forma espectral. Se considera que el intervalo en el que se promedian los periodos de interés es entre $0.2 \mathrm{~T}_{\mathrm{e}}$ y $1.3 \mathrm{~T}_{\mathrm{e}}$, de acuerdo con la especificación de las NTC-S2017, donde $\mathrm{T}_{\mathrm{e}}$ es el periodo fundamental de la estructura. El objetivo de seleccionar la medida de intensidad es buscar que todos los movimientos sísmicos tengan una propiedad similar en un intervalo de periodos que permita reducir la dispersión en la respuesta estructural para cada una de las intensidades a la que se escalan los movimientos. Como ejemplo, en la figura $6 \mathrm{~b}$ se muestran los espectros de respuesta de los diez acelerogramas seleccionados y escalados a una intensidad promedio $\mathrm{Sa}_{\text {prom }} / \mathrm{g}=0.46$. En la figura $6 \mathrm{~b}$ también se muestran el promedio de los espectros escalados, y el espectro de diseño (ver figura 4).

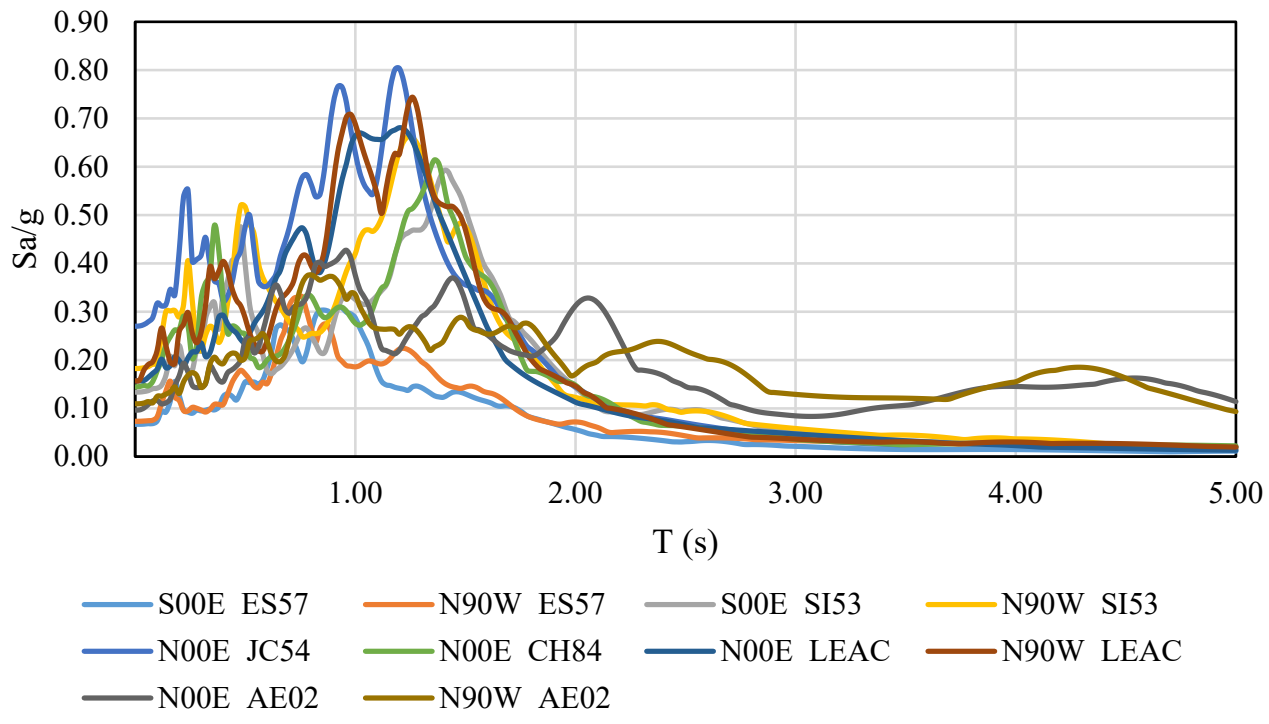

Figura 6a. Espectros elásticos de seudo-aceleración 

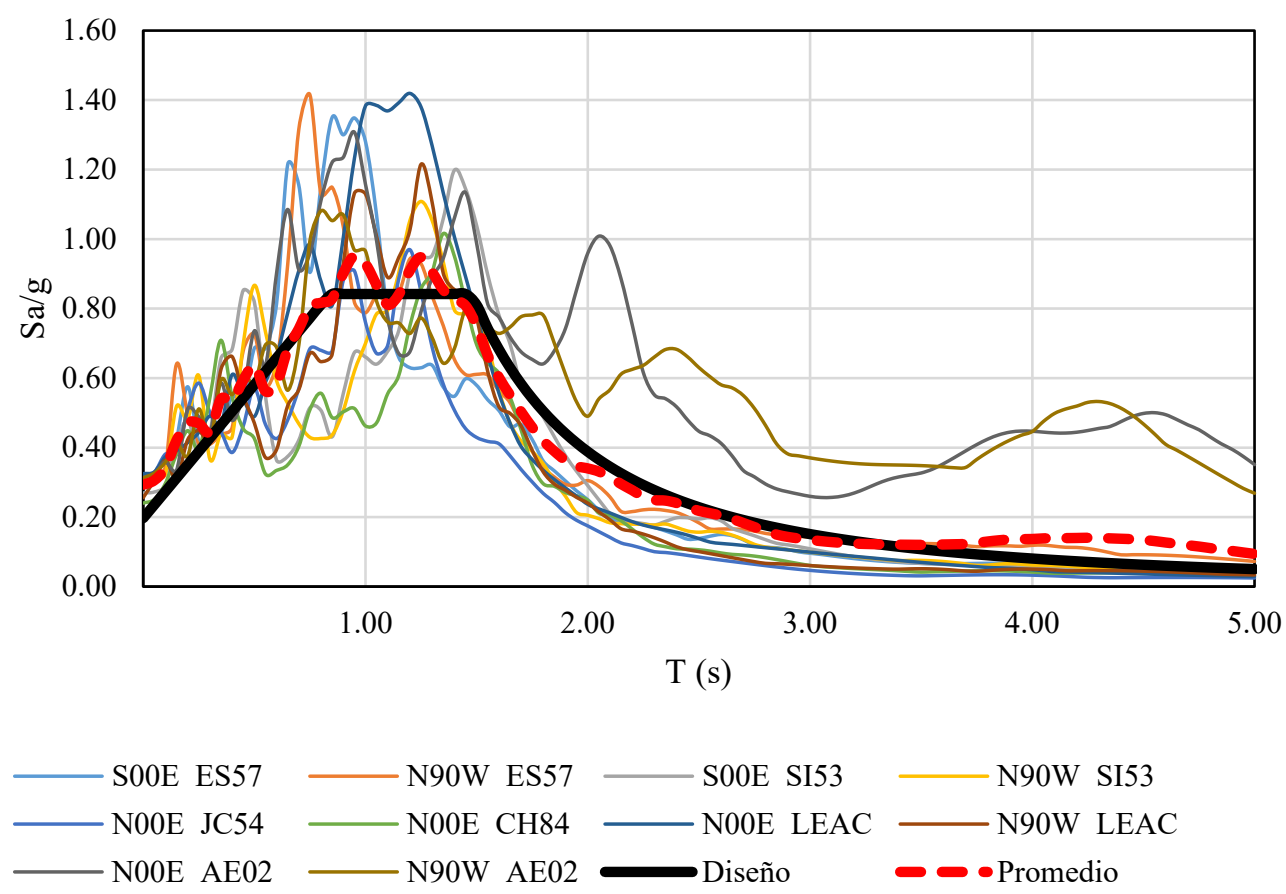

Figura 6b. Espectros de respuesta escalados a una intensidad promedio $\mathrm{Sa}_{\text {prom }} / \mathrm{g}=0.46$

\section{CURVAS DE FRAGILIDAD}

La función de fragilidad se define como la probabilidad condicional de igualar o exceder un determinado valor de demanda estructural, dado un valor de intensidad. La función de fragilidad se define por la ecuación 2, donde se asume que la función de distribución condicional de la demanda estructural es de tipo lognormal (Rosenblueth y Esteva, 1972).

$P(D>d \mid I M=i m)=1-\Phi\left(\frac{\ln (y / \widehat{D})}{\sigma_{\ln D}}\right)$

donde:

$P(D>d \mid I M=i m)$ : es la probabilidad condicional de exceder un valor $d$, dado que se presentó una intensidad $I M=i m$.

$\Phi$ : es la distribución de probabilidad normal estándar.

$\ln (y / \widehat{D})$ : es el logaritmo natural de la intensidad, $y$, entre la mediana de la demanda estructural, $\widehat{D}$. $\sigma_{l n D}$ : es la desviación estándar del logaritmo natural de la demanda estructural.

Los resultados se expresan aquí en términos de curvas de fragilidad. Estas permiten estimar la probabilidad de exceder cierto valor de distorsión máxima de entrepiso (DME), dado un nivel de pseudoaceleración promedio $\mathrm{Sa}_{\text {prom. }}$. La demanda estructural corresponde a la distorsión máxima de todos los entrepisos, la cual se obtiene de análisis dinámicos no lineales, correspondientes a los diez movimientos que se listan en la tabla 5.

Las curvas de fragilidad sísmica generalmente se obtienen para el desplazamiento o distorsión máxima de la azotea (HAZUS, 2013; Liu et al., 2016); sin embargo, en el caso de edificios irregulares en 
su altura estos parámetros no son adecuados. Choudhury y Kaushik (2018) muestran que la máxima demanda ocurre en el nivel en donde se encuentra la irregularidad, y en el caso de edificios con planta baja débil, esta ocurre en el entrepiso bajo (lo que es de esperarse); es decir, estos autores muestran que, en este caso, es más eficiente y realista considerar como parámetro de demanda una respuesta local que una global.

En el presente estudio se hace ver que efectivamente el edificio original con planta baja débil (caso S1) presenta la máxima demanda estructural en su piso bajo débil, ante cualquier nivel de intensidad sísmica (alto o bajo); sin embargo, los edificios rehabilitados (casos S2 y S3) presentan su máxima demanda de entrepiso en el entrepiso bajo (marcos de concreto reforzado) cuando las estructuras se someten a intensidades sísmicas relativamente bajas, pero la demanda máxima se presenta en el primer entrepiso (muros de mampostería) cuando se someten a intensidades altas. En el presente estudio las curvas de fragilidad corresponden al estado límite de seguridad de vidas (asociado a intensidades sísmicas altas), y pueden corresponder ya sea al entrepiso bajo (marco de concreto) o al primer entrepiso (muros de mampostería), según sea el caso en estudio.

Lo anterior hace ver que se debe hacer una selección adecuada del parámetro de demanda de ingeniería, de manera que represente de forma realista el comportamiento estructural, especialmente en edificios con irregularidad vertical.

\section{MODELACIÓN DE LAS ESTRUCTURAS}

Los análisis no lineales se realizaron con el programa de cómputo Ruaumoko 3D (Carr, 2000). Para ello, se generó un modelo tridimensional de elementos tipo "barra" para vigas y columnas, el cual permite asignar comportamiento no-lineal concentrado debido a la flexión de sus extremos. Para los muros se utilizó el método de la columna ancha modificado (Teran-Gimore et al., 2009) donde los muros se modelan con elementos tipo "resorte" en el que se permite asignar una rótula plástica por elemento con comportamiento histéretico debido a cortante. Las principales consideraciones para el modelado no-lineal son las siguientes: en las columnas se consideró la interacción de la carga axial con la flexión en dos direcciones; en las vigas se consideró la flexión en el eje principal; en ambos casos se utilizó el modelo de Takeda modificado, que toma en cuenta la degradación de rigidez y resistencia con las siguientes consideraciones: 1) para el caso S1: en las columnas se considera una envolvente bilineal sin pendiente post elástica y se toma una ductilidad local máxima de 1.5 debido al bajo detallado de refuerzo transversal; para vigas se considera un modelo trilineal con una pendiente post-elástica del 10\% hasta alcanzar el $\mathrm{M}_{\mathrm{Rmax}}$, una ductilidad local máxima de 13 y una resistencia residual del 10\%; 2) para los casos S2 y S3: en las columnas se considera una envolvente trilineal con pendiente post-elástica del $10 \%$ hasta alcanzar el $\mathrm{M}_{\mathrm{Rmax}}$, se toma una ductilidad local máxima igual a 6 para el caso S2, y de 3 para el caso S3; en ambos casos se consideró el nivel de carga axial en las columnas y una resistencia residual del 20\% (ASCE/SEI 41-17); para las vigas se tomaron las mismas consideraciones que las del caso S1. El comportamiento no lineal en los muros de mampostería se localizó al centro de las columnas anchas con una falla predominante en cortante, para el modelado de la degradación de su rigidez y de su resistencia se utilizó un modelo tri-lineal el cual se calibró usando información de pruebas cíclicas realizadas en México por Flores y Alcocer (1996); en la figura 7 se muestra uno de sus resultados experimentales, así como la regla de comportamiento (SINA modificado degradante - Ruaumoko 3D) que se ajustó en el presente estudio. Para las diagonales de pandeo restringido se utilizó un modelo bilineal con pendiente post-elástica positiva igual a 4\% (Sues et al., 1988). 


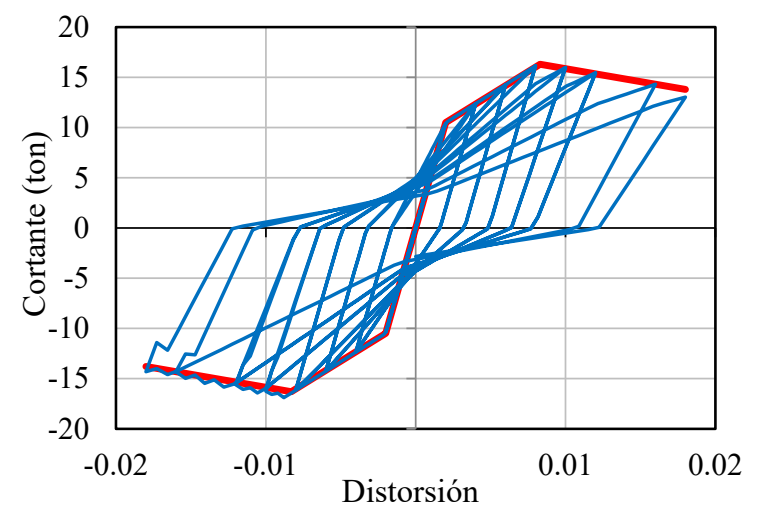

a. Modelo SINA modificado degradante

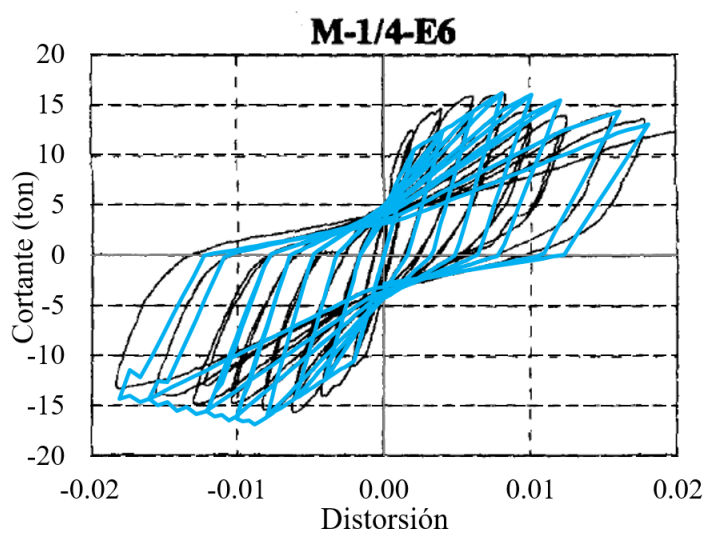

b. Comprobación del ajuste con prueba experimental

Figura 7. Comparación de la respuesta experimental (Flores y Alcocer, 1996) con la analítica

\section{RESULTADOS}

Los resultados de los análisis dinámicos incrementales (ADI) se expresan en términos de curvas de capacidad (Vamvatsikos y Cornell, 2002; Montiel y Ruiz, 2007), las cuales son útiles para determinar la intensidad que genera cierto nivel de distorsión máxima en el sistema estructural, así como la capacidad del mismo sistema. Es importante hacer notar que las curvas ADI no representan lo mismo que las curvas obtenidas de un análisis estático no lineal ("push over"). Este último relaciona la fuerza cortante que experimenta el sistema en su base (o en un entrepiso) y el desplazamiento máximo de azotea (o de entrepiso); mientras que la curva ADI relaciona el potencial destructivo de un sismo representado por una medida de intensidad del movimiento sísmico (que en el presente estudio es $M I=S a_{\text {prom }}$ ) y un parámetro de respuesta estructural (que en el presente estudio es la distorsión máxima de entrepiso). El agotamiento de capacidad en una curva ADI se ve reflejado de dos formas: 1) como una interrupción repentina de la curva que se caracteriza por una inestabilidad numérica, 2) como una línea horizontal (o casi horizontal) que indica el agotamiento de la capacidad de deformación. Para ver con mayor claridad las diferencias entre las curvas antes mencionadas (resultantes de ADI y de "push-over"), el lector puede recurrir a la referencia de Montiel y Ruiz (2007), quienes presentan una comparación entre curvas correspondientes a un análisis dinámico incremental (ADI) y las correspondientes curvas resultantes de un análisis estático no lineal ("push-over") de edificios de varios niveles y crujías.

Para desarrollar las curvas ADI se realizaron análisis dinámicos incrementales para los movimientos que se listan en la tabla 5, considerando un intervalo de intensidades espectrales promedio entre $\mathrm{Sa}_{\mathrm{prom}}=0.1$ y $0.8 \mathrm{~g}$, con incrementos de $0.01 \mathrm{~g}$.

Existen diferentes planteamientos para determinar la capacidad de deformación última de los sistemas estructurales a través de un análisis detallado de la curva ADI (Vamvatsikos y Cornell, 2002); en el presente trabajo se acepta que las máximas deformaciones que pueden desarrollar los sistemas estructurales son las distorsiones máximas permisibles $\left(\gamma_{\text {máx }}\right)$ que se especifican en las tablas 4.2.1 y 4.2.3 de las NTC-S2017. En lo que sigue se realiza una evaluación de la capacidad estructural de los tres casos en estudio. 


\section{Comportamiento del caso $\mathrm{S} 1$ ante distintas intensidades sísmicas}

La figura 8 muestra las curvas de capacidad del edificio S1, con línea más gruesa se muestra el promedio de los diez análisis y con color gris el resultado de cada uno de los ADI. Analizando la curva gruesa roja, se puede ver que la distorsión máxima $\gamma_{\text {máx }}=0.015$ se alcanza a una intensidad $(\mathrm{Sa} / \mathrm{g})$ aproximadamente de 0.35 (ver líneas punteadas azules), también se puede notar que, a partir de ese valor, las distorsiones máximas crecen significativamente sin que exista un incremento sustancial en el valor de la intensidad. Se recuerda al lector que, según las NTC-S2017, una estructuración de marcos de concreto reforzado de baja ductilidad tiene una distorsión máxima permisible igual a $\gamma_{\text {máx }}=0.015$.

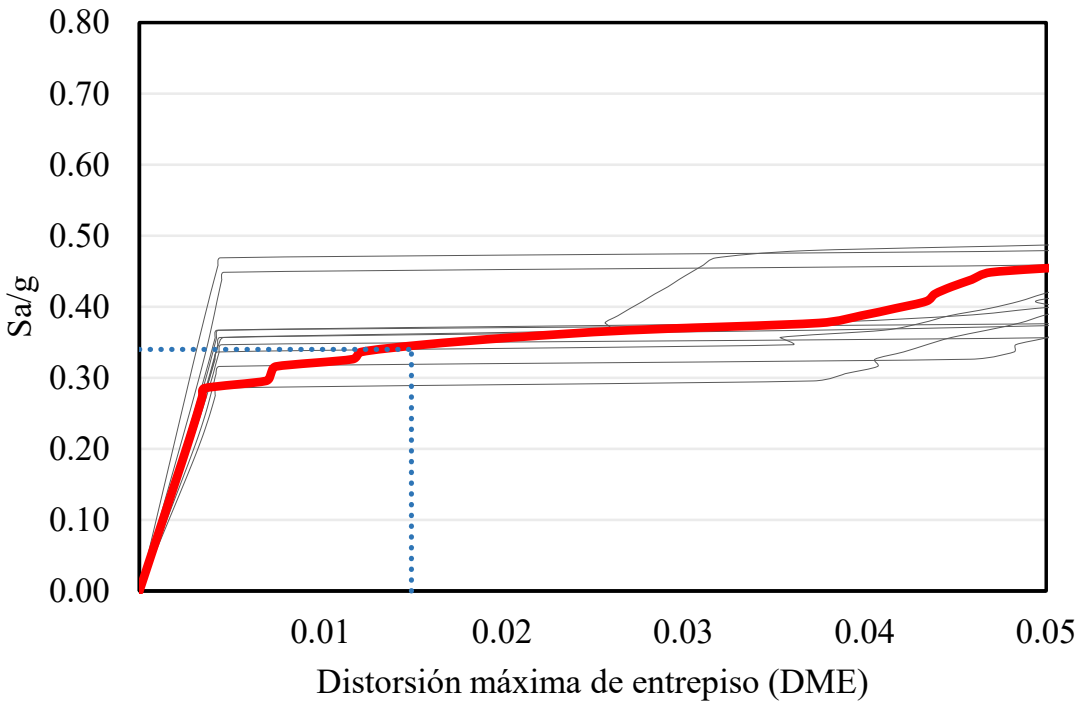

Figura 8. Curvas ADI correspondientes al caso S1

Las figuras 9a, 9b, 9c, 9d, 9e y 9f muestran las distorsiones máximas de entrepiso del caso S1 para intensidades de $0.1 \mathrm{~g}, 0.2 \mathrm{~g}, 0.28 \mathrm{~g}, 0.3 \mathrm{~g}, 0.34 \mathrm{~g}$ y $0.40 \mathrm{~g}$, respectivamente. En las figuras se muestra con línea punteada roja la distorsión máxima promedio de entrepiso correspondiente a los diez análisis dinámicos, y con línea gris el resultado de cada uno de los análisis. En las figuras $9 \mathrm{a}$ a $9 \mathrm{~d}$ se puede ver que para intensidades bajas las mayores distorsiones máximas ocurren en la planta baja, y que al incrementar la intensidad no existe un aumento sustancial en las distorsiones máximas de los entrepisos superiores, que se mantienen por debajo de 0.010 . Por otro lado, la figura 9e muestra que para una intensidad de $0.34 \mathrm{~g}$ se alcanza una distorsión máxima promedio igual a $\gamma=0.015$, que corresponde a la distorsión máxima tolerable que establecen las NTC-S2017 para marcos de concreto de baja ductilidad; también se puede ver que la distorsión máxima del primer entrepiso $(\mathrm{PB})$ de la figura 9e corresponde con el punto en la curva de capacidad intersectado por las líneas punteadas de la figura 8 , e indica la condición para la que los desplazamientos crecen de forma significativa sin un incremento significativo de la intensidad. Finalmente, la figura 9f muestra las distorsiones de entrepiso para una intensidad de $0.4 \mathrm{~g}$, en donde se observa que la distorsión de máxima de entrepiso de planta baja es mayor a 0.04 , lo que representa falla del sistema estructural.

Las figuras 10a y 10b muestran las curvas de histéresis de una columna y de un muro para una intensidad de $0.34 \mathrm{~g}$, respectivamente. En las figuras se puede ver que la columna (figura 10a), ubicada en el eje 3 y B de la planta baja, alcanza su máxima capacidad y deja de tomar carga, mientras que el muro (figura 10b), que se localiza en el eje 4 entre $\mathrm{C}$ y D del segundo entrepiso, permanece con comportamiento elástico lineal. 


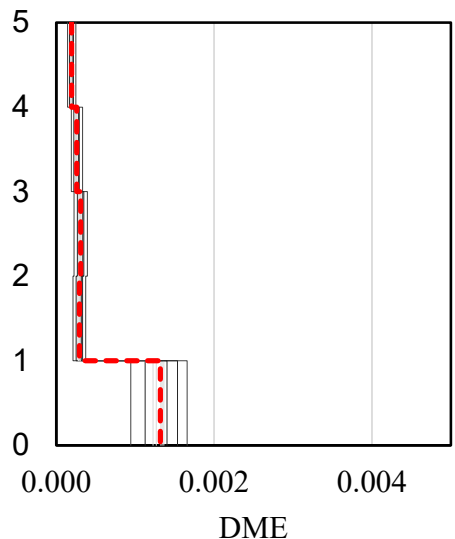

a) $0.10 \mathrm{~g}$

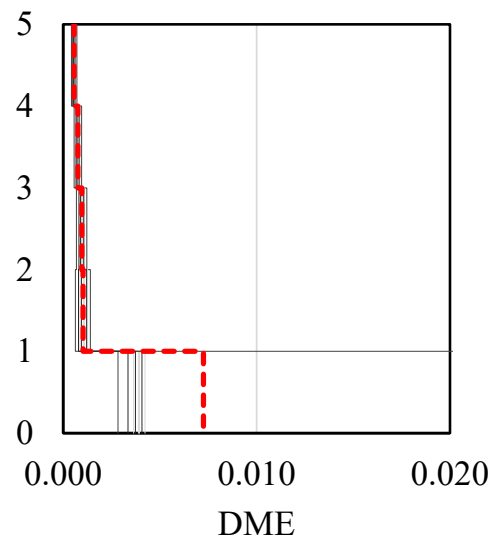

d) $0.30 \mathrm{~g}$

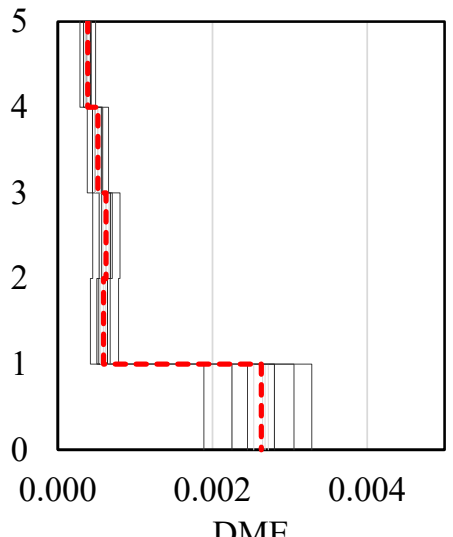

b) $0.20 \mathrm{~g}$

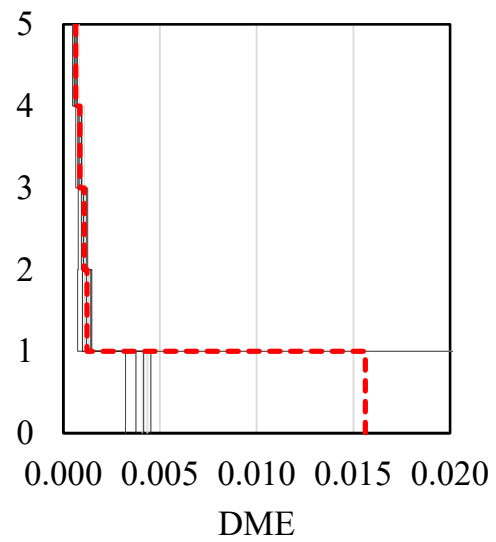

e) $0.34 \mathrm{~g}$

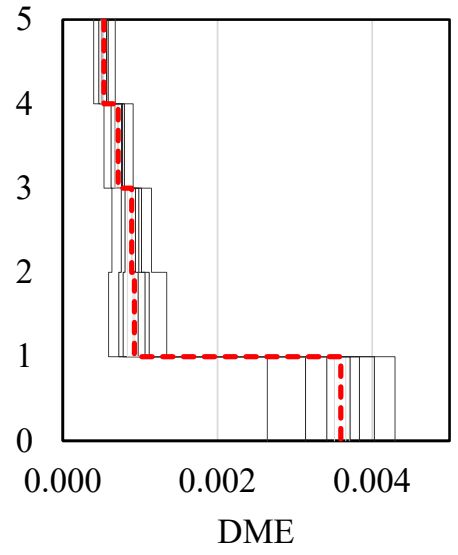

c) $0.28 \mathrm{~g}$

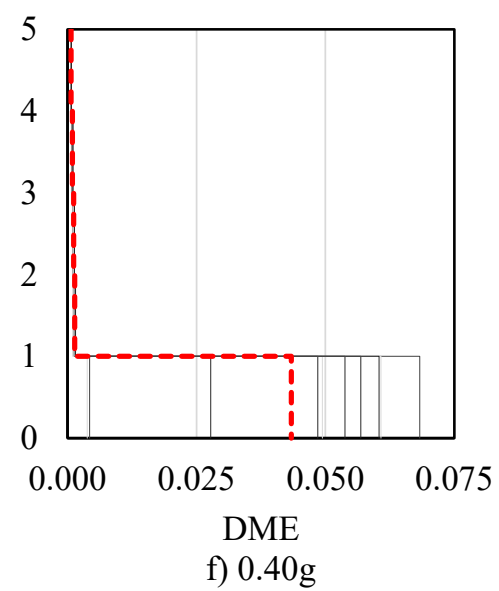

Figura 9. Distorsiones máximas de entrepiso del caso S1, para diferentes valores de intensidad

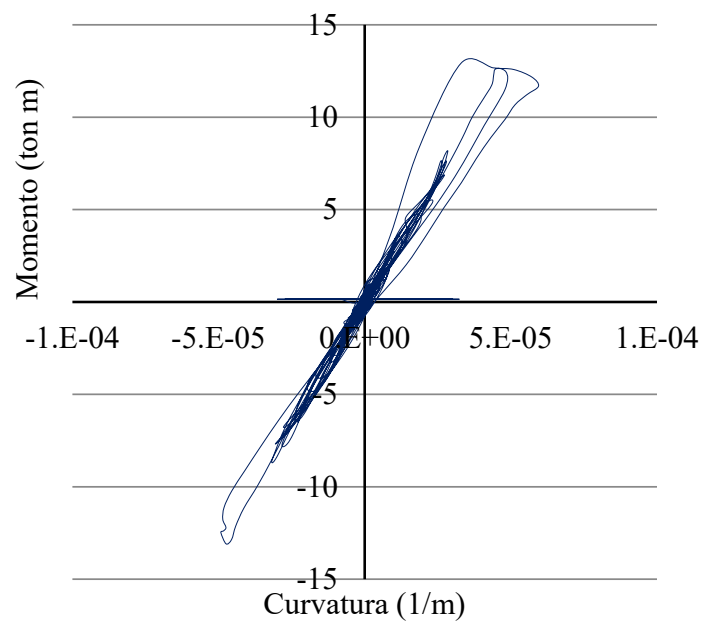

a) Curvas de histéresis de la columna ubicada en el eje 3 y B de la planta baja

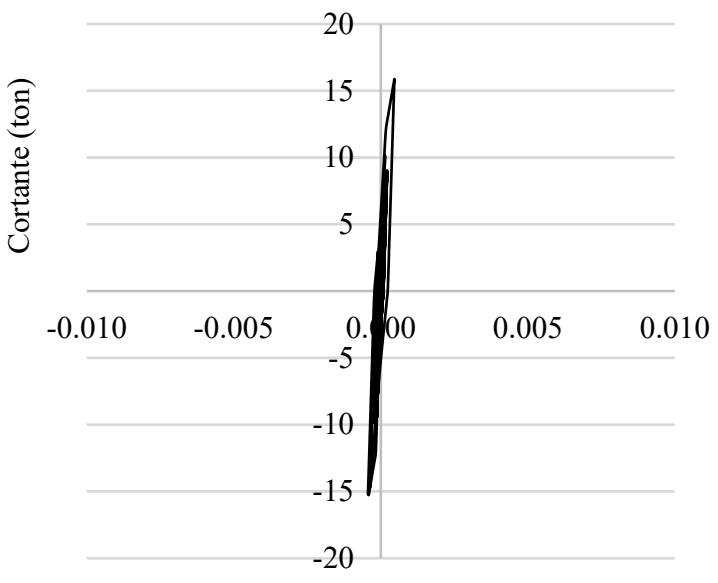

Distorsión

b) Curvas de histéresis del muro del eje 4 entre $\mathrm{C}$ y D del segundo entrepiso

Figura 10. Curvas de histéresis de muro y columna para el caso $\mathrm{S} 1$ para una intensidad $\mathrm{Sa} / \mathrm{g}=0.34$ 


\section{Comportamiento de los casos S2 y S3 ante distintas intensidades sísmicas}

De la misma forma que para el caso S1, se obtuvieron resultados de ADI para los casos S2 y S3. Más adelante se muestra que la máxima distorsión puede localizarse en un entrepiso diferente a la planta baja.

Las figuras 11 y 12 muestran las curvas ADI correspondientes a los casos S2 y S3, respectivamente. Estas curvas corresponden a la distorsión máxima de todos los entrepisos de los edificios rehabilitados. Más adelante se explica en qué entrepiso ocurre la distorsión máxima, lo que depende del nivel de intensidad sísmica al que se somete la estructura. Las figuras 11 y 12 muestran que los casos S2 y S3 tienen una capacidad estructural similar. Esto se debe a que desde el proceso de diseño se buscó que tanto la resistencia global como la rigidez del caso S3 fuesen similares a las del caso S2. También se puede apreciar que las intensidades que generan distorsiones superiores a 0.010 son $58 \%$ más grandes que las correspondientes al edificio S1; es decir, los casos S2 y S3 presentan una resistencia lateral 1.6 veces mayor a la del caso S1.

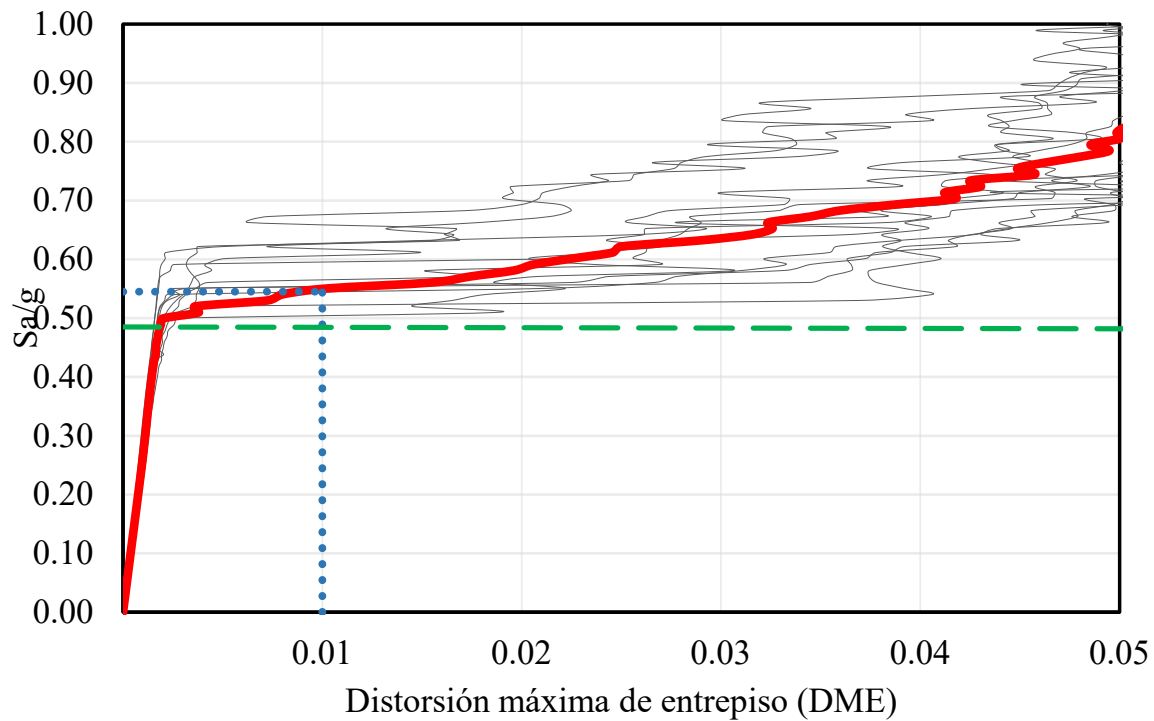

Figura 11. Curvas ADI correspondientes al caso S2

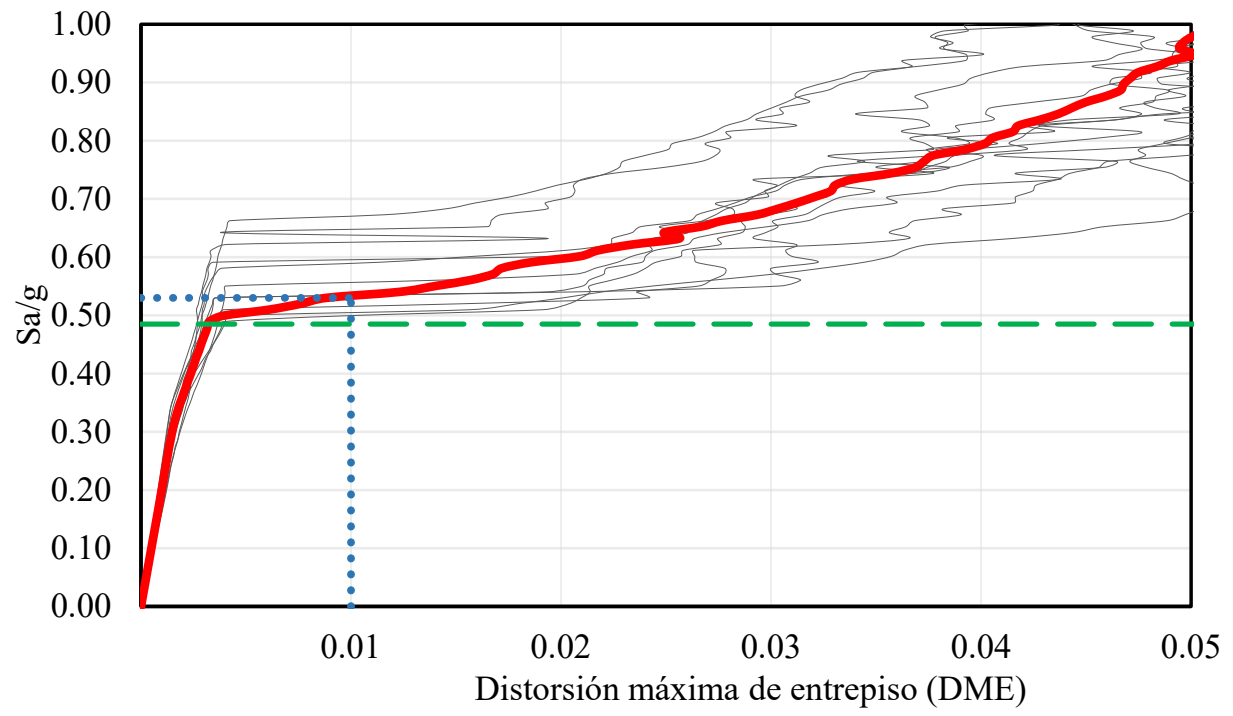

Figura 12. Curvas ADI correspondientes al caso S3 
Con el fin de evaluar el comportamiento de los edificios en términos de las distorsiones máximas de entrepiso (DME) que se alcanzan en los casos S2 y S3 ante distintas intensidades, es conveniente realizar un análisis detallado, correlacionándolos con algunos puntos de las curvas figuras 11 y 12.

En las figuras 13 a 21 se muestran las distorsiones máximas de entrepiso (DME) correspondientes a diferentes intensidades. Estas figuras muestran el comportamiento de los tres edificios en estudio: a) caso $\mathrm{S} 1, \mathrm{~b}$ ) caso $\mathrm{S} 2$ y c) caso S3; en este último se muestra, además, el ciclo de histéresis del CRP (en unidades de toneladas, $\mathrm{t}, \mathrm{y}$ metros, $\mathrm{m}$ ) asociado al movimiento sísmico que da lugar a distorsiones máximas de entrepiso cercanas a las distorsiones máximas promedio de entrepiso.

Las figuras 13 y 14 corresponden a DME para una intensidad $\mathrm{Sa} / \mathrm{g}=0.10$ y 0.20 , respectivamente. En ellas se puede ver que la distorsión máxima promedio se mantiene por debajo de 0.001 para los casos S2 y S3, mientras que el caso S1 muestra una distorsión máxima elevada en la PB. Las figuras 13c y 14c muestran, en la esquina superior derecha, que el comportamiento de los CRP es elástico lineal.

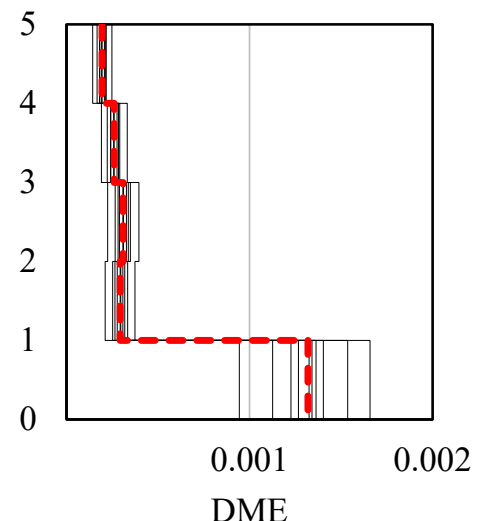

(a) Caso S1

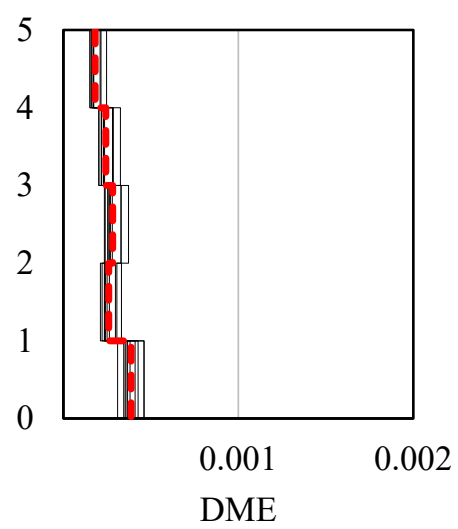

(b) Caso S2

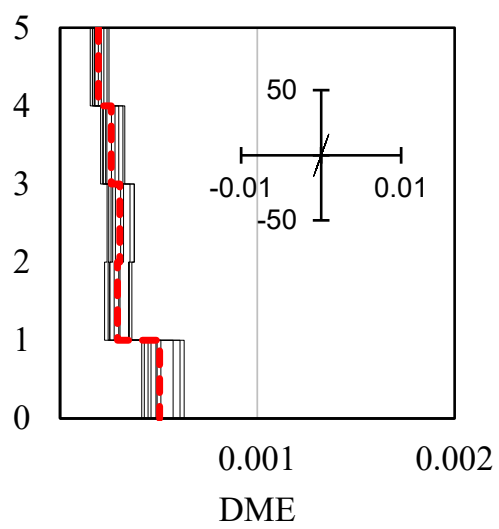

(c) Caso S3

Figura 13. Perfil de distorsiones para $\mathrm{Sa} / \mathrm{g}=0.10$; ciclo histerético en t y $\mathrm{m}$

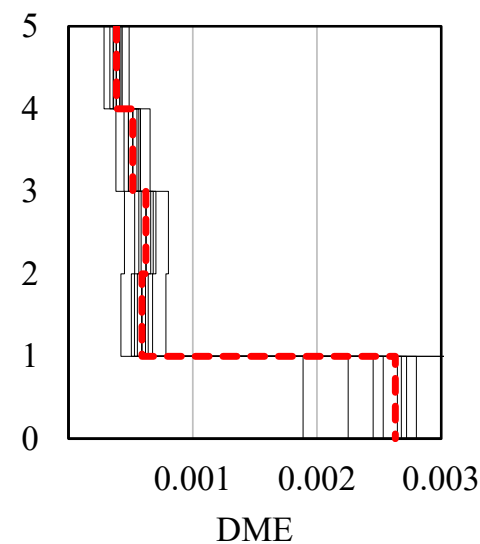

(a) Caso S1

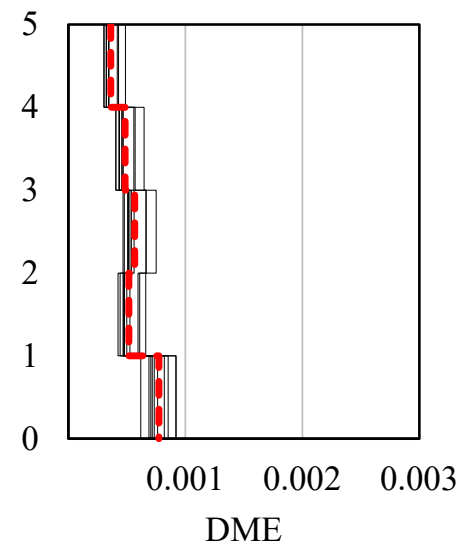

(b) Caso S2

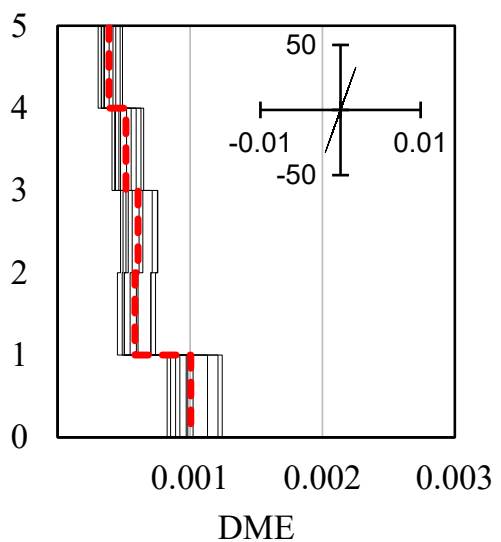

(c) Caso S3

Figura 14. Perfil de distorsiones para $\mathrm{Sa} / \mathrm{g}=0.20$; ciclo histerético en $\mathrm{t}$ y $\mathrm{m}$

En la figura 15, correspondiente a una intensidad de $0.3 \mathrm{~g}$, se observa que los casos S2 y S3 tienen distorsiones máximas promedio de entrepiso cercanas a 0.001 ; sin embargo, para el caso S1 (figura 15a) las 
DME crecen a más del doble en la PB. La figura 15 c muestra que a partir de esta intensidad $(\mathrm{Sa} / \mathrm{g}=0.3)$ inicia el comportamiento inelástico del CRP.

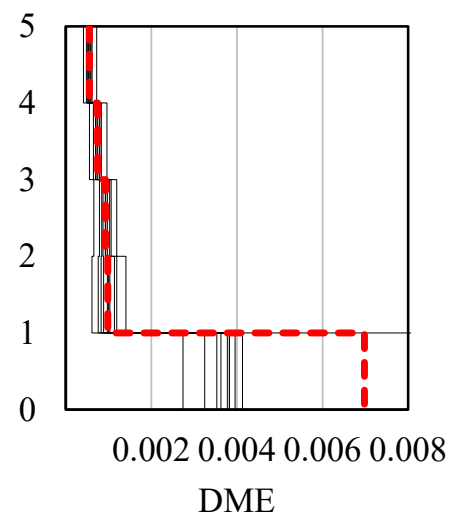

(a) Caso S1

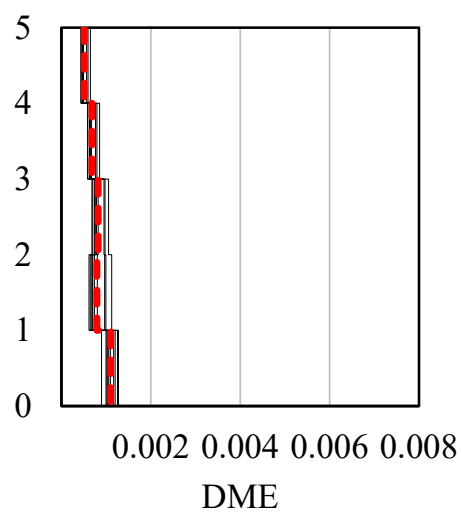

(b) Caso S2

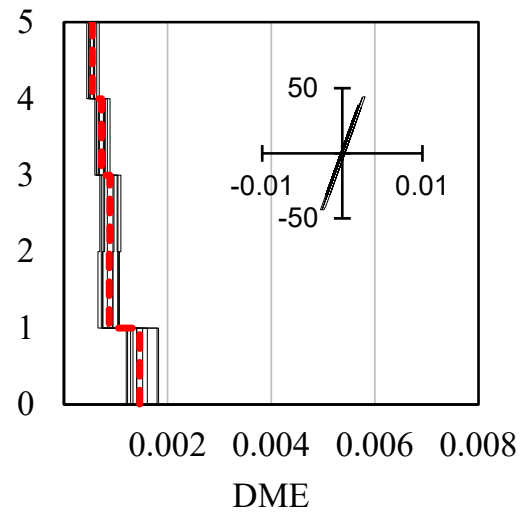

(c) Caso S3

Figura 15. Perfil de distorsiones para $\mathrm{Sa} / \mathrm{g}=0.30$; ciclo histerético en $\mathrm{t}$ y $\mathrm{m}$

La figura 16a muestra que para una intensidad $\mathrm{Sa} / \mathrm{g}=0.41$ la distorsión máxima promedio de entrepiso del caso S1 es 0.043 , valor que es 2.8 veces mayor al límite máximo permisible por las NTCS2017. Por otro lado, en las figuras $16 \mathrm{~b}$ y $16 \mathrm{c}$ se puede apreciar que la distorsión máxima promedio de entrepiso correspondiente a los modelos S2 y S3, están por debajo de 0.003. Se distingue que en el caso S2 (figura 16b) las distorsiones máximas correspondientes a algunos movimientos sísmicos son mayores que las correspondiente a la planta baja, lo que indica que las fuerzas se están redistribuyendo en los entrepisos superiores. En la figura 16c se puede ver el comportamiento bilineal de uno de los CRP.

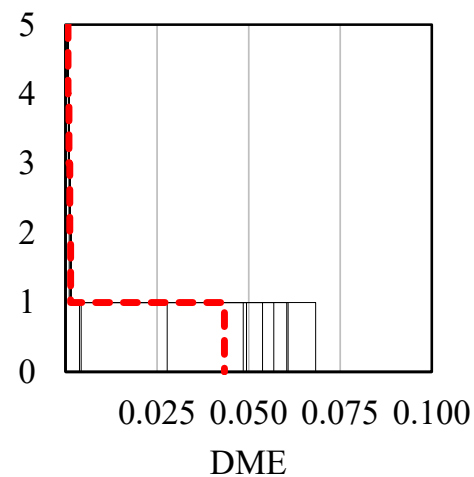

(a) Caso S1

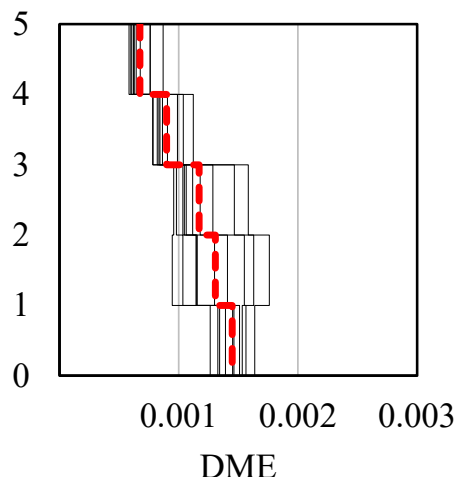

(b) Caso S2

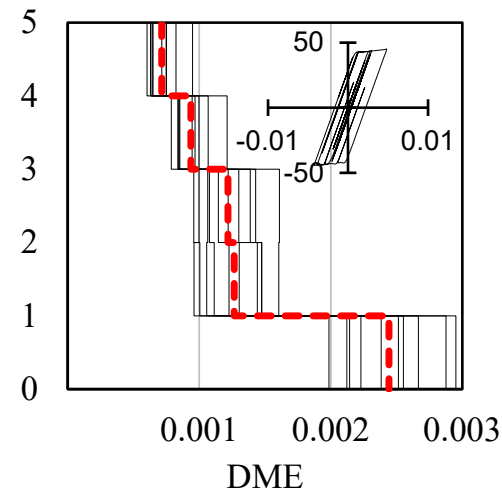

(c) Caso S3

Figura 16. Perfil de distorsiones para $\mathrm{Sa} / \mathrm{g}=0.41$; ciclo histerético en $\mathrm{t}$ y $\mathrm{m}$

En las figuras 17,18 y 19 se presentan los perfiles de DME correspondientes a intensidades $\mathrm{Sa} / \mathrm{g}=$ $0.49,0.50$ y 0.51 , respectivamente. Para el caso S2 a partir de una intensidad de $\mathrm{Sa} / \mathrm{g}=0.49$ las distorsiones máximas de la planta baja se mantienen por debajo de 0.002 y no tienen un incremento importante a intensidades mayores. Se hace notar que, para el mismo caso, a partir de $\mathrm{Sa} / \mathrm{g}=0.49$, la $\mathrm{DME}$ se presenta en el primer entrepiso y no en la planta baja. Esto se indica en la figura 11 con una con una línea verde horizontal interrumpida. En esta figura se puede apreciar que el sistema estructural presenta un comportamiento global casi lineal hasta $\mathrm{Sa} / \mathrm{g}=0.49$. Por otro lado, en el caso S3 las distorsiones máximas en la planta baja se mantienen por debajo de 0.004. En este caso, la distorsión máxima de entrepiso se presenta en el primer entrepiso (y no en la planta baja) a partir de $\mathrm{Sa} / \mathrm{g}=0.51$. Esto se indica en la figura 12 
con una línea verde horizontal interrumpida, en donde se puede apreciar que, para este nivel de intensidad sísmica, el sistema estructural rehabilitado con CRP presenta un comportamiento global no lineal.

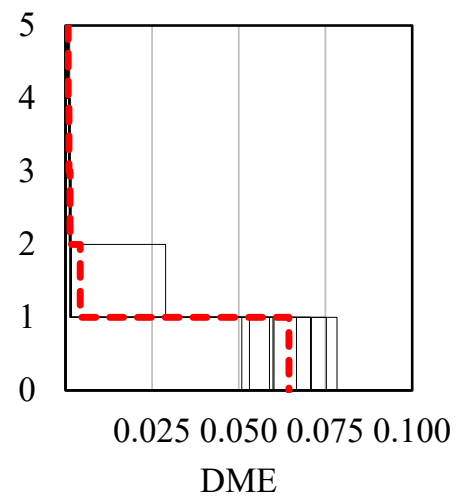

(a) Caso S1

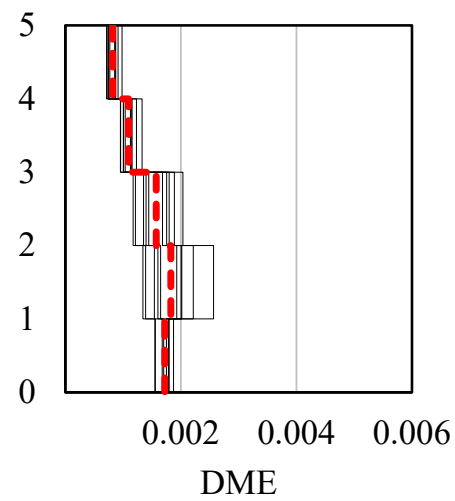

(b) Caso S2

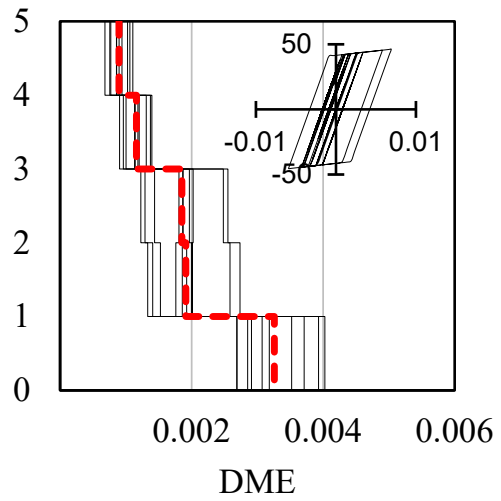

(c) Caso S3

Figura 17. Perfil de distorsiones para $\mathrm{Sa} / \mathrm{g}=0.49$; ciclo histerético en $\mathrm{t}$ y $\mathrm{m}$

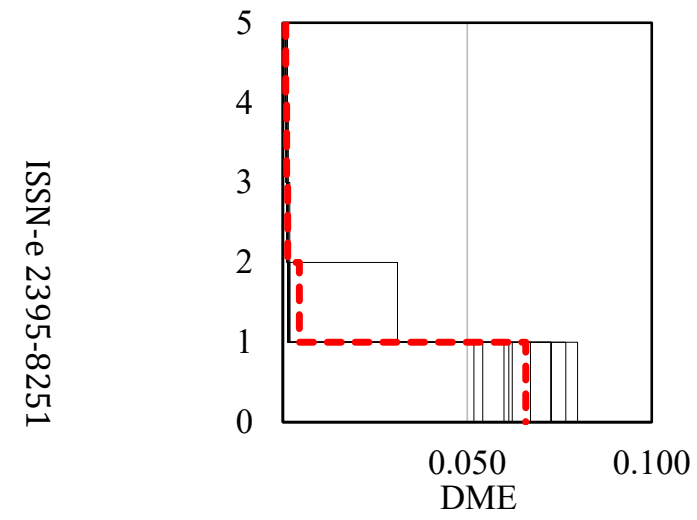

(a) Caso $\mathrm{S} 1$

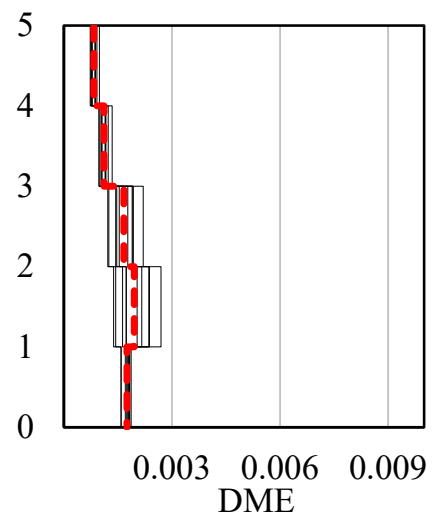

(b) Caso S2

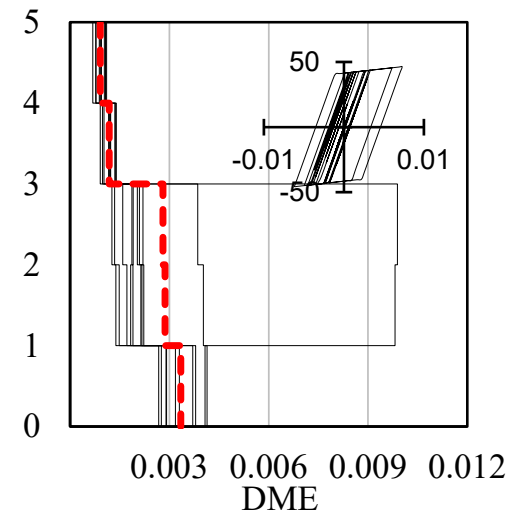

(c) Caso S3

Figura 18. Perfil de distorsiones para $\mathrm{Sa} / \mathrm{g}=0.50$; ciclo histerético en t y $\mathrm{m}$

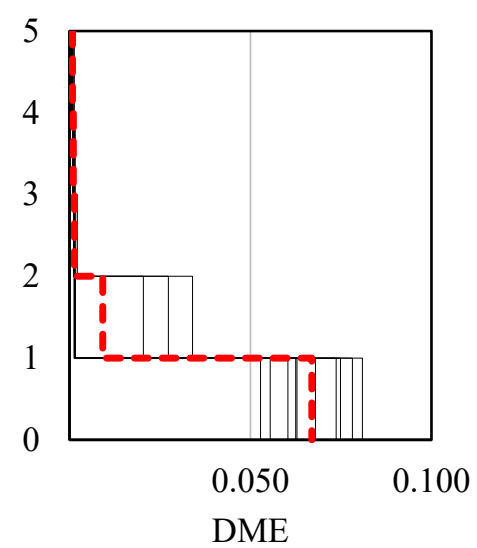

(a) Caso $\mathrm{S} 1$

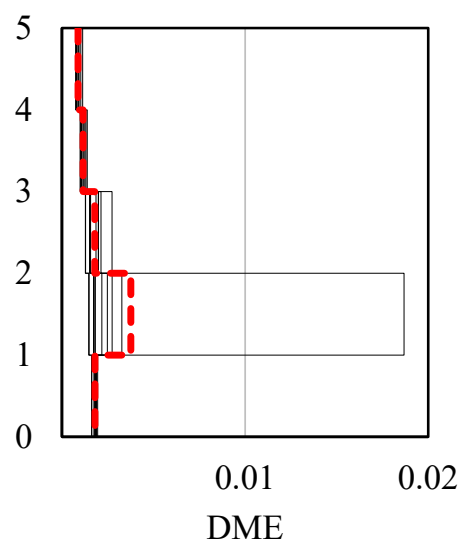

(b) Caso S2

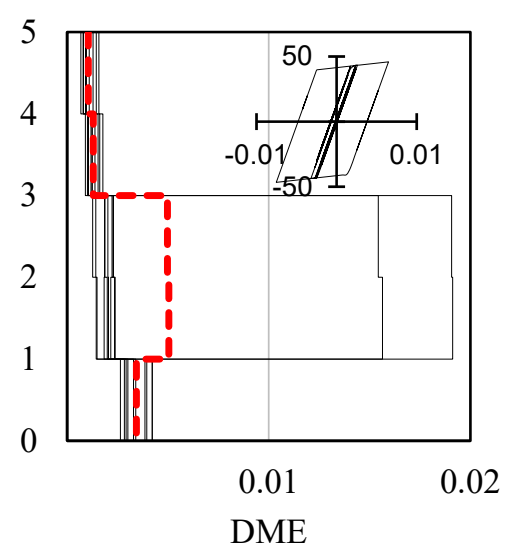

(c) Caso S3

Figura 19. Perfil de distorsiones para $\mathrm{Sa} / \mathrm{g}=0.51$; ciclo histerético en $\mathrm{t}$ y $\mathrm{m}$ 
La figura 20 muestra el perfil de DME para una intensidad de $0.53 \mathrm{~g}$. En la figura $20 \mathrm{~b}$ se observa que las distorsiones máximas promedio del segundo entrepiso (línea punteada roja) están por debajo de 0.01 (valor de diseño máximo permisible según las NTC-S2017), mientras que para el edificio S3 (figura 20c) la distorsión máxima promedio del segundo entrepiso es de 0.010 , lo que indica que los muros ubicados entre el primero y el segundo nivel desarrollaron la máxima distorsión de entrepiso que permite la normatividad para muros de mampostería confinada de piezas macizas con acero de refuerzo horizontal suministrado por acero en las juntas o mallas de alambre $\left(\gamma_{\text {máx }}=0.010\right)$. Lo anterior es congruente con lo que se muestra en linea punteada azul en la curva IDA de la figura 12. Por otro lado, en la figura 20c se puede apreciar que la ductilidad local desarrollada por el CRP es aproximadamente igual a 3 . De forma similar, en la figura $21 \mathrm{~b}$ se puede ver que la máxima distorsión reglamentaria $\left(\gamma_{\text {máx }}=0.010\right)$ en los muros de mampostería del segundo entrepiso se alcanza para un intensidad de $\mathrm{Sa} / \mathrm{g}=0.55$. Estos dos valores $\left(\gamma_{\text {máx }}\right.$ y $\mathrm{Sa} / \mathrm{g}$ ) corresponden a la intersección de las lineas azules punteadas de la figura 11.

Las figuras $22 \mathrm{a}, \mathrm{b}$ y c muestra, como ejemplo, el comportamiento histerético de un muro del caso S2 y del S3, para diferentes niveles de intensidad y diferentes movimientos sísmicos. En estas figuras se puede apreciar que, aunque el valor promedio de la distorsión máxima de entrepiso que se muestra en las figuras 20 y 21 se mantiene dentro de los valores permisibles indicados en las NTC-S2017, la respuesta y el deterioro de la rigidez y la resistencia de los muros para cada movimiento, puede ser muy diferente. Por otro lado, en estos dos casos (S2 y S3) las columnas y vigas en planta baja no presentan comportamiento inelástico.

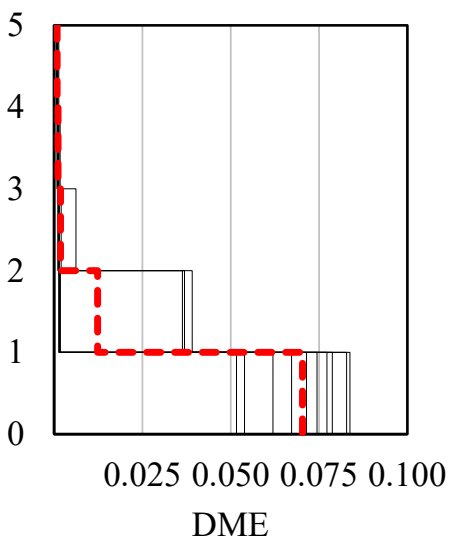

(a) Caso S1

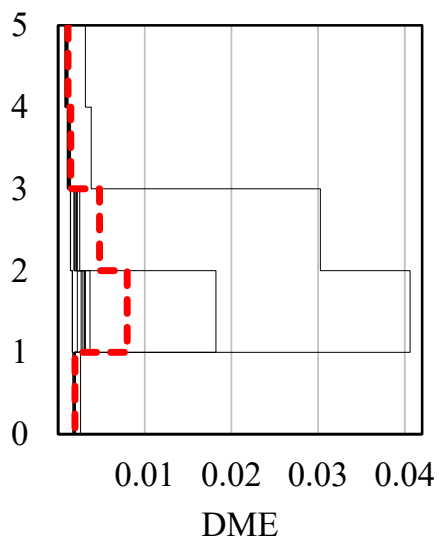

(b) Caso S2

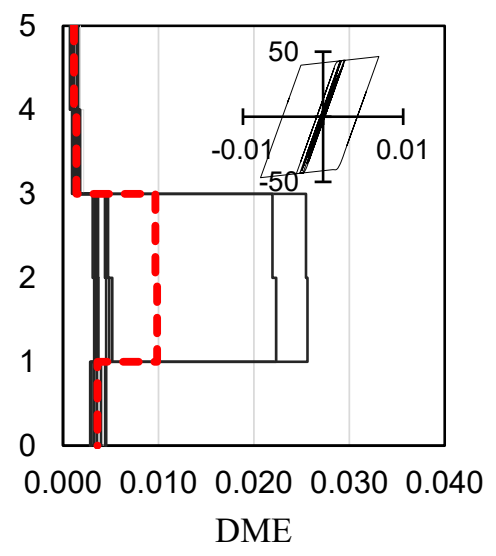

(c) Caso S3

Figura 20. Perfil de distorsiones para $\mathrm{Sa} / \mathrm{g}=0.53$; ciclo histerético en t y $\mathrm{m}$

Las curvas ADI que se presentan en las figuras 8,11 y 12 junto con los perfiles de DME de las figuras 9, y 13 a 21 son útiles para conocer la capacidad y la respuesta de las estructuras; sin embargo, cuando las estructuras se someten a movimientos sísmicos con altas intensidades se obtiene gran dispersión en las respuestas estructurales debido al comportamiento no lineal de las estructuras, como es de esperarse. Por ejemplo, la figura $21 \mathrm{~b}$ muestra que, aunque todas las señales se escalaron a una intensidad espectral promedio de $0.55 \mathrm{~g}$, existen distorsiones máximas en el segundo entrepiso que van desde 0.002 hasta 0.04 , dependiendo del movimiento sísmico que se trate. Es decir, en un análisis (asociado a cierto movimiento sísmico) la DME es tan pequeña que puede considerarse que no hay daños en los elementos estructurales; sin embargo, para otro movimiento sísmico la respuesta excede en cuatro veces la distorsión máxima permisible de entrepiso. 


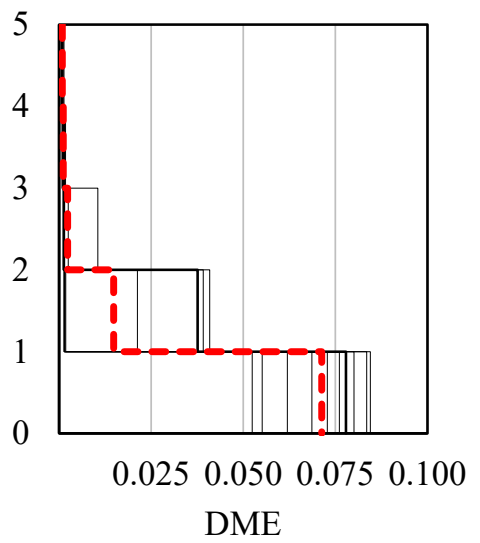

(a) Caso S1

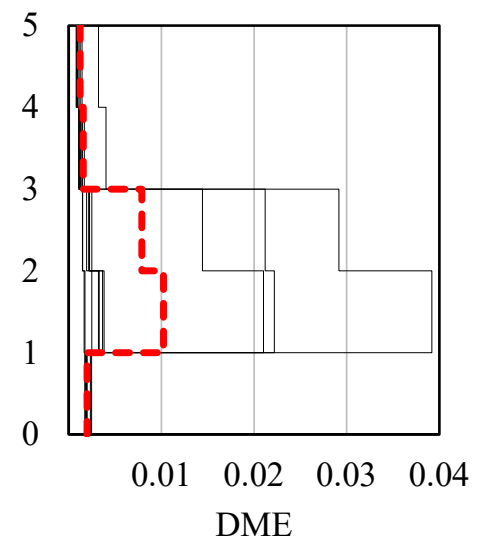

(b) Caso S2

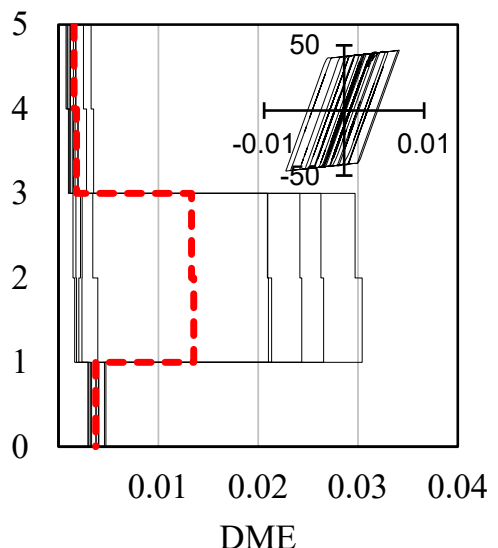

(c) Caso S3

Figura 21. Perfil de distorsiones para $\mathrm{Sa} / \mathrm{g}=0.55$; ciclo histerético en t y $\mathrm{m}$

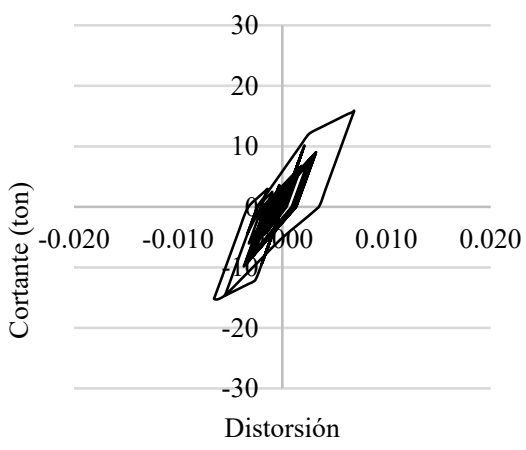

(a) Caso S2 para una intensidad $\mathrm{Sa} / \mathrm{g}=0.55$ del movimiento 2 .

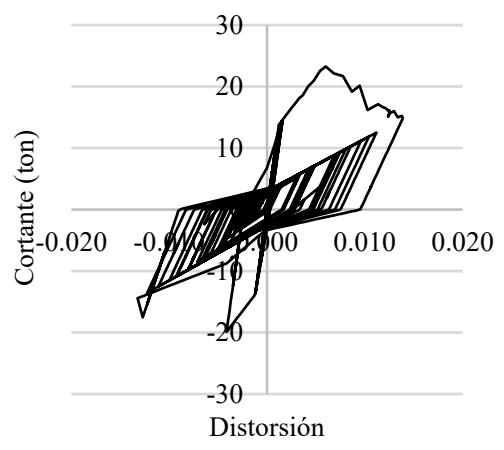

(b) Caso S2 para una intensidad $\mathrm{Sa} / \mathrm{g}=0.55$ del movimiento 3 .

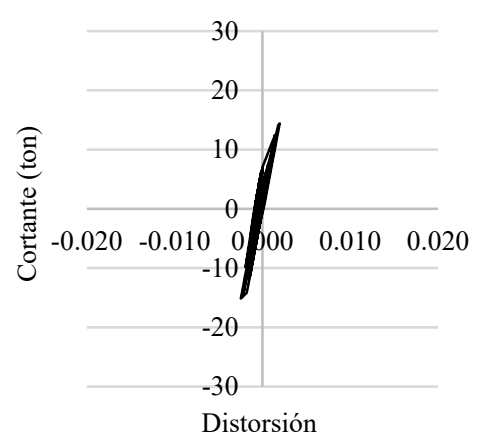

(c) Caso S3 para una intensidad $\mathrm{Sa} / \mathrm{g}=0.53 \mathrm{del}$ movimiento 1 .

Figura 22. Curvas de histéresis del muro ubicado en el segundo entrepiso en el eje 4 entre ejes C y D

En las figuras $13 \mathrm{~b}$ a $17 \mathrm{~b}$ correspondientes al caso 2, se puede ver que para intensidades $\mathrm{Sa} / \mathrm{g}$ iguales o menores que 0.49 , la mayor distorsión siempre ocurre en la planta baja. Para estos casos la desviación estándar del logaritmo de la demanda estructural, $\sigma_{\ln D}$, es en promedio de aproximadamente 0.15 ; $\sin$ embargo, para intensidades altas $(\mathrm{Sa} / \mathrm{g} \geq 0.50)$, que corresponden a las figuras $18 \mathrm{~b}$ a $21 \mathrm{~b}$, la mayor DME ocurre en el segundo entrepiso. En estas figuras se puede apreciar que la dispersión es mayor a medida que la intensidad aumenta. En las figuras $23 \mathrm{a}$, b y c se muestra el valor de $\sigma_{l n D}$ versus la intensidad para los casos S1, S2 y S3, respectivamente.

Con el fin de tomar en cuenta la variabilidad de la respuesta estructural, enseguida se obtienen las curvas de fragilidad sísmica que consideren los parámetros estadísticos (mediana y desviación estándar del logaritmo) de la respuesta estructural (ver ecuación 2). 


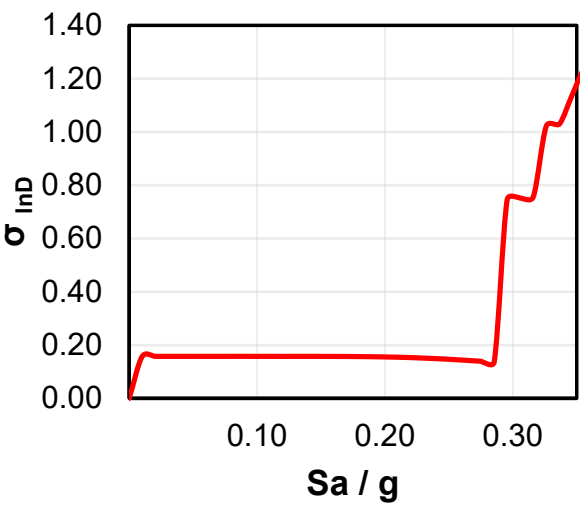

a) Caso S1

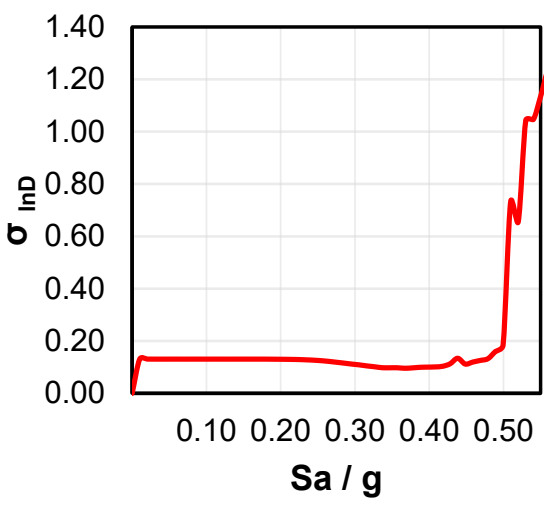

b) Caso S2

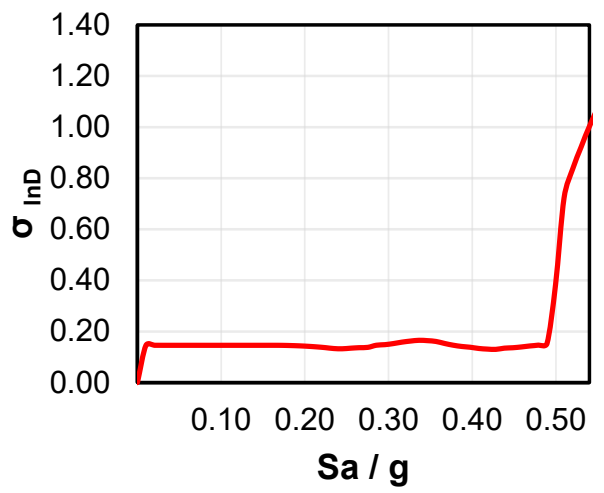

c) Caso S3

Figura 23. Desviación estándar del logaritmo de la DME

\section{Curvas de fragilidad}

La figura 24 muestra dos curvas de fragilidad correspondientes al PB del caso S1, para dos límites de distorsión máxima. La primera (línea discontinua) corresponde a una distorsión de $\mathrm{d}=0.004$, asociada al estado límite de servicio, mientras que la línea continua corresponde a $\mathrm{d}=0.015$ (asociada al estado límite de seguridad contra colapso).

De la figura 8 se puede deducir que para el caso S1, la deformación máxima de entrepiso asociada al colapso incipiente (que corresponde a un valor de distorsión máxima promedio de 0.015) ocurre a una intensidad $\mathrm{Sa} / \mathrm{g}=0.34$. Cuando se selecciona este valor de intensidad en el eje horizontal de la figura $24, \mathrm{y}$ se interseca verticalmente la curva de fragilidad (línea continua) asociada con una distorsión máxima de entrepiso $\mathrm{d}=0.015$, se obtiene una probabilidad de exceder dicha distorsión igual a 32 por ciento.

Por otro lado, la figura 25 muestra las curvas de fragilidad de los casos S1, S2 y S3 para las máximas distorsiones tolerables asociadas al estado límite de prevención del colapso que especifican las NTC-S2017. Estos valores son iguales a 0.015 para los marcos de concreto reforzado (planta baja), e iguales a 0.01 para los muros de mampostería confinada de piezas macizas con acero de refuerzo horizontal suministrado por acero en las juntas o mallas de alambre. 


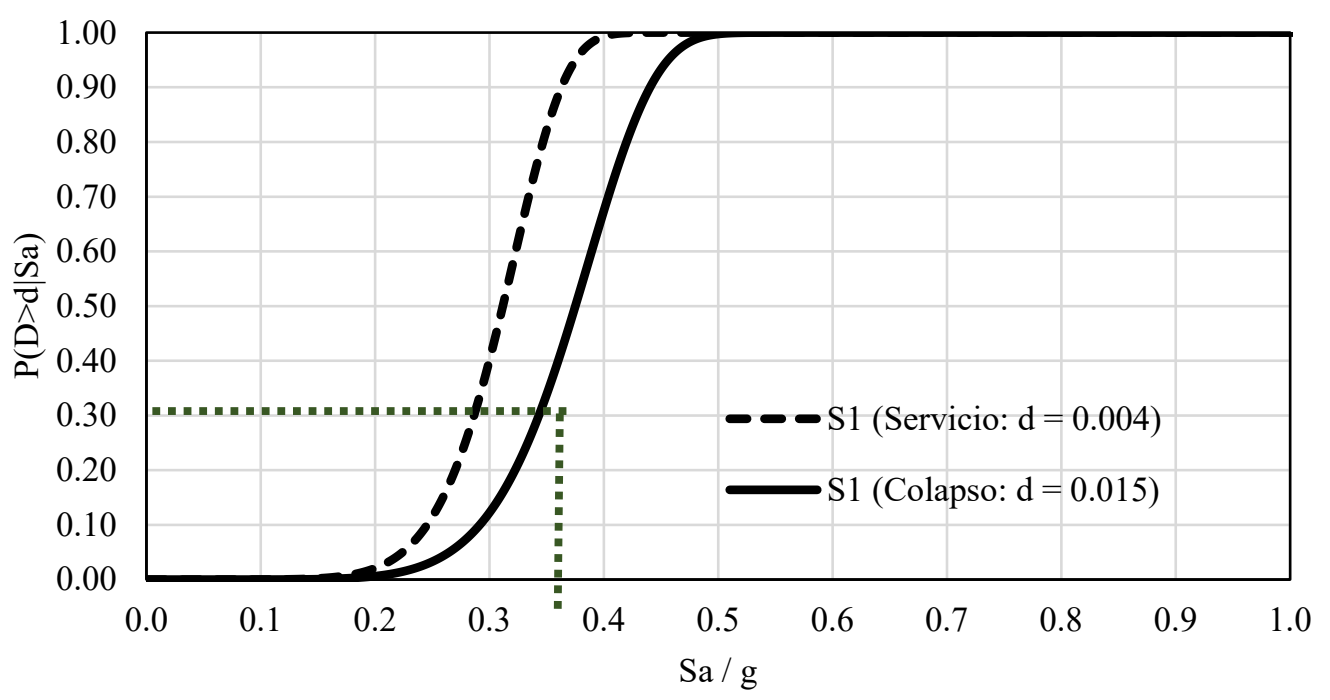

Figura 24. Curvas de fragilidad correspondientes al piso bajo (de concreto reforzado) del caso S1

La figura 25 muestra que para una intensidad tanto de $0.55 \mathrm{~g}$ como de $0.53 \mathrm{~g}$ (ver figuras 11 y 12) la probabilidad de exceder una distorsión máxima $\mathrm{d}=0.015$ en la PB del edificio original (caso S1) es de 100 por ciento; sin embargo, la probabilidad de exceder una distorsión máxima $\mathrm{d}=0.10$ en los muros de mampostería del segundo entrepiso de los edificios rehabilitados (casos S2 y S3) es de 30 por ciento (ver línea interrumpida de la figura 22).

En la figura 25 se puede verificar que el caso S3 (edificio con encamisado de acero y CRP) presenta una fragilidad estructural similar a la del caso S2 (edificio con encamisado de concreto) correspondiente al estado límite de colapso, cuando se considera como parámetro de demanda estructural la distorsión máxima de entrepiso, con lo que se ha cumplido con el objetivo del presente estudio.

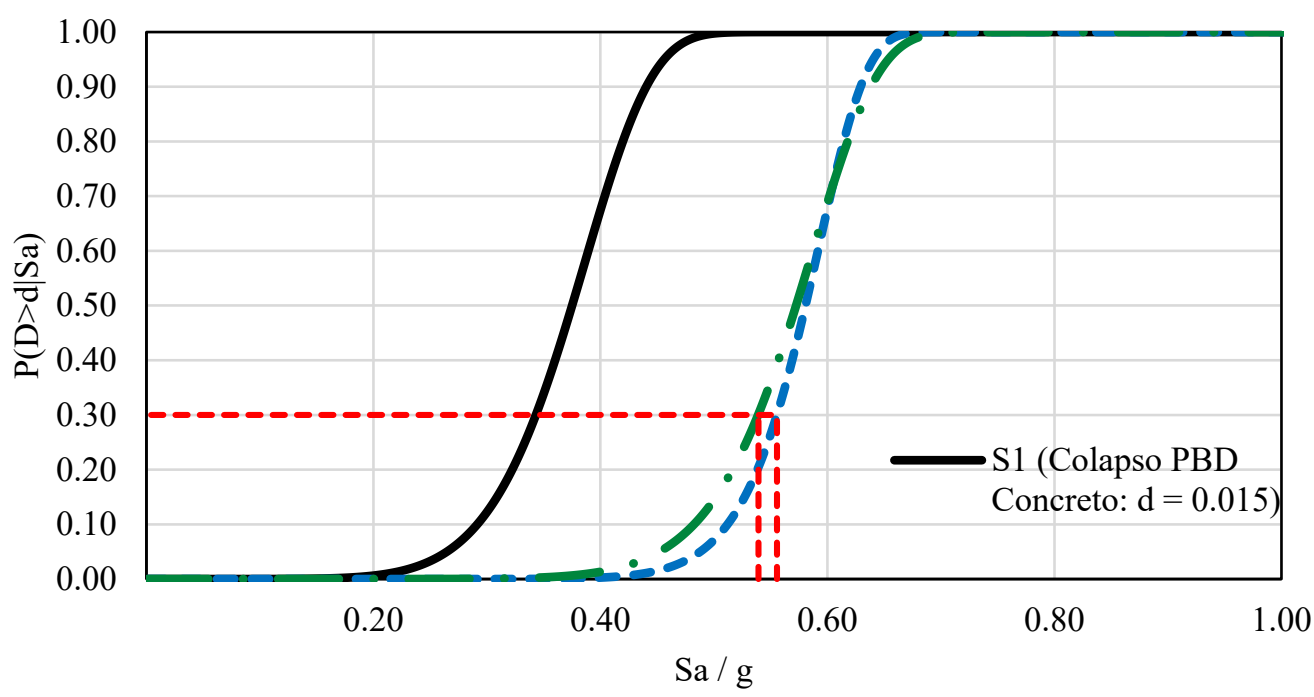

Figura 25. Curva de fragilidad. Seguridad contra colapso. Casos S1, S2 y S3 


\section{CONCLUSIONES}

Se analizó el comportamiento sísmico y se evaluó la fragilidad estructural de tres edificios:

S1: Edificio original con planta baja débil (PBD) en donde no se satisfacen las condiciones del RCDF2004, por lo que el edificio presenta problemas de planta baja débil. Se hace la observación de que si se hubieran respetado las NTC-C2004, el edificio no requeriría ser rehabilitado (Cabrera López y Ruiz Gómez, 2019).

S2: Edificio rehabilitado en las columnas de su planta baja, mediante la técnica de encamisado de concreto reforzado, y además malla electrosoldada y aplanado a los muros de mampostería confinada de los niveles superiores; $\mathrm{y}$

S3: Edificio rehabilitado en las columnas de su planta baja con encamisado de acero más contravientos restringidos al pandeo (CPR), más malla electrosoldada y aplanado en los muros de mampostería confinada.

Para el análisis se utilizaron diez movimientos sísmicos registrados en la zona de transición de la Ciudad de México el 19 de septiembre de 2017, que es la zona en donde ocurrió el colapso de varios edificios de mediana altura con piso bajo débil.

Se encontró que el valor más grande de la distorsión máxima de entrepiso (DME) del edificio original sin rehabilitar (caso S1) se presentó siempre en el piso bajo, independientemente de la intensidad del movimiento sísmico (intensidad baja o alta).

Después de rehabilitar el edificio original, se verificó que los edificios S2 y S3 no se encuentran dentro de la clasificación de "edificio con planta baja débil", según la definición que proporcionan las NTCS-2017.

Se encontró que cuando los edificios rehabilitados (casos S2 y S3) se sometieron a movimientos sísmicos con una intensidad $(\mathrm{Sa} / \mathrm{g})$ aproximadamente igual o menor a la que provoca la fluencia del sistema estructural, la distorsión máxima de entrepiso (DME) se presentó en la PB; sin embargo, cuando se supuso una intensidad más grande que esta, la DME migró al siguiente (segundo) entrepiso.

Se demostró que las técnicas de rehabilitación S2 y S3 presentan curvas de fragilidad sísmica similares. Si el caso S3 se hubiera rehabilitado siguiendo las especificaciones de las NTC-S2107 (suponiendo $\mathrm{Q}=4$ ) el área transversal de los CRP había sido menor (Santos et al, 2017), lo que conduciría a una fragilidad más grande que la correspondiente al caso S2 (Jiménez Jordán, 2018; Ruiz et al, 2019); sin embargo, el objetivo principal del presente estudio es demostrar que la técnica de rehabilitación que utiliza CRP es tan confiable como la solución tradicional a base de encamisado de concreto.

La solución de encamisar las columnas de la PB (caso S2) implica hacer trabajos de re-cimentación de la estructura, no usar el estacionamiento durante el tiempo que se hagan estos trabajos y el encamisado de columnas, así como tener incomodidad temporal en el espacio de acceso al edificio. Por otro lado, la solución que utiliza CRP (caso S3) es menos invasiva, algunos lugares de estacionamiento pasarían a tener otro uso, y el costo inicial de los CRP es mayor que el encamisado de columnas de concreto; sin embargo, no hay necesidad de re-cimentar la estructura, ya que las fuerzas que se transmiten a la cimentación se reducen con respecto a las correspondientes al caso 2 (Ruiz et al., 2019).

En casos como el estudiado aquí, normalmente la decisión de elegir una u otra solución de 
rehabilitación es de los dueños del inmueble (cliente), quienes deberán basar su decisión no solo en el costo inicial de rehabilitación estructural, sino en un análisis de costos totales de la estructura, que considere los posibles daños que provoquen los movimientos sísmicos que se espera que se presenten en el sitio, durante la vida útil del edificio. El costo total implica considerar costos de rehabilitación, posible pérdida de vidas, lesionados, grado de invasión de cada uno de los sistemas de rehabilitación, rapidez en la rehabilitación o sustitución de elementos estructurales, pérdida de espacios, etc.

\section{AGRADECIMIENTOS}

El presente estudio se realizó dentro de los proyectos PAPIIT IN103517 e IN100320 de la DGAPA UNAM. Se agradece al CIRES haber proporcionado los registros sísmicos que se utilizaron en este estudio. Los autores agradecen los valiosos comentarios de dos revisores anónimos, quienes contribuyeron a enriquecer el documento. Los coautores (segundo, tercero y cuarto) agradecen al CONACyT el apoyo económico brindado durante sus estudios de posgrado.

\section{REFERENCIAS}

ASCE (2017), "Seismic evaluation and retrofit of existing buildings", ASCE Standard, ASCE/SEI 41-17, Reston, Virginia, USA. DOI: 10.1061/9780784414859

Baker, J y A Cornell (2006). "Spectral shape, epsilon and record selection", Earthquake Engineering and Structural Dynamics, Vol. 35 pp. 1077-1095. DOI: 10.1002/eqe.571

Beigi, H A, C Christopoulos, T Sullivan y G M Calvi (2014), "Gapped-inclined braces for seismic retrofit of soft-story buildings", ASCE Journal of Structural Engineering, Vol. 140, No. 11. DOI: 10.1061/(ASCE)ST.1943-541X.0001006

Cabrera López, J L y S E Ruiz Gómez (2019), "Fragilidad de un edificio con piso bajo débil diseñado con las versiones del RCDF-1976, -1987, -2014 y -2017”, XXII Congreso Nacional de Ingeniería Sísmica, Monterrey, N. L., México.

Carr, A (2011), Ruaumoko 3D, inelastic dynamic analysis program, University of Catenbury, Departament of Civil Engineering.

Chopra, A K, D P Clough y R W Clough (1973), "Earthquake resistance of buildings with a soft fist story", Earthquake Engineering \& Structural Dynamics, Vol. 1, No. 4, pp. 347-355. DOI: $\underline{10.1002 / \text { eqe } 4290010405}$

Choudhury, T y H B Kaushik (2018), "Component level fragility estimation for vertically irregular reinforced concrete frames", Journal of Earthquake Engineering. DOI: $\underline{10.1080 / 13632469.2018 .1453413}$

CIRES, Base de datos del Centro de Instrumentación y Registro Sísmico, A. C. http://www.cires.org.mx/

Esteva, L (1992), "Nonlinear seismic response of soft-fist.-story buildings subjected to narrow-band accelerograms", Earthquake Spectra, Vol. 8, No. 3, pp. 373-389. DOI: 10.1193/1.1585686 
Flores, L y S Alcocer (1996), "Calculated response of confined masonry structures", 11th World Conference on Earthquake Engineering. Acapulco, México, Paper No. 1830. ISBN: 0080428223

Gobierno del Distrito Federal (2004), "Normas técnicas complementarias para diseño de estructuras de concreto", Gaceta Oficial del Distrito Federal. https://data.consejeria.cdmx.gob.mx/portal old/uploads/gacetas/Octubre04 06 103BISTI.pdf

Gobierno del Distrito Federal (2004), "Normas técnicas complementarias para diseño de estructuras de mampostería", Gaceta Oficial del Distrito Federal. https://data.consejeria.cdmx.gob.mx/portal_old/uploads/gacetas/Octubre04_06_103BISTI.pdf

Gobierno del Distrito Federal (2004), "Normas técnicas complementarias para diseño por sismo", Gaceta Oficial del Distrito Federal. https://data.consejeria.cdmx.gob.mx/portal_old/uploads/gacetas/Octubre04_06 103BISTII.pdf

Gobierno de la Ciudad de México (2017), "Normas técnicas complementarias para diseño por sismo", Gaceta Oficial de la Ciudad de México. https://data.consejeria.cdmx.gob.mx/portal_old/uploads/gacetas/841541814eb94e624a8b5c1d07b3c823. $\underline{\mathrm{pdf}}$

Guerrero, H, Ji T y J A Escobar (2016a), "Experimental studies of a steel frame model with and without buckling-restained braces", Revista de Ingeniería Sísmica, No. 95, pp. 33-52. DOI: $\underline{10.18867 / \text { ris. } 95.338}$

Guerrero, H, Ji T, A Terán-Gilmore y J A Escobar (2016b), “A method for preliminary seismic design and assessment of low-rise structures protected with buckling-restrained braces", Engineering Structures, Vol. 123, pp. 141-154. DOI: 10.1016/j.engstruct.2016.05.015

HAZUS (2013) HAZUS - MH MR5, Advanced Engineering Building Module (AEBM), Technical and User's Manual, FEMA, Washington, D. C.

Hernández García, D A y A Tena Colunga (2016), "Estudio paramétrico de modelos representativos de estructuras propensas a desarrollar pisos suaves ante excitaciones sísmicas de suelos blandos”, Revista de Ingeniería Sísmica, No. 95, pp. 53-80. DOI: 10.18867/ris.95.405

Jiménez Jordán, R (2018), "Curvas de fragilidad de un edificio tipo con planta baja débil dañado por el sismo S-19/2017, y rehabilitado con contravientos restringidos al pandeo", Tesis de Maestría, Programa de Maestría y Doctorado en Ingeniería, UNAM, México.

Liu, X, Z-Y Wu y F Liang (2016), "Multidimensional performance limit state for probabilitic seismic demand analysis", Bulletin of Earthquke Engineering, Vol. 4, No. 4, pp. 415-443. DOI: 10.1007/s10518-016-0013-6

Miyamoto, H y R Scholl (1996), "Case study: seismic rehabilitation of a non-ductile soft story concrete structure using viscous dampers", 11th World Conference on Earthquake Engineering, Acapulco, México, Paper No. 315. ISBN: 0080428223

Montiel, M A y S E Ruiz (2007), "Influence of structural capacity uncertainty on seismic reliability of 
buildings under narrow-band motions", Earthquake Engineering and Structural Dynamics, Vol. 36, pp. 1915-1934. DOI: $10.1002 /$ eqe. 711

Pérez-Gavilán, J J (editor) (2015), Guía de análisis de estructuras de mampostería, Sociedad Mexicana de Ingeniería Estructural, Comité de Mampostería. ISBN- 978-607-95994-1-6

Rosenblueth, E y L Esteva (1972). "Reliability basis for some Mexican codes". ACI Publ. SP-31 1972, 31, pp. 1-41.

Ruiz, S E (2019), Comentarios al Apéndice B (edificios con disipadores de energía sísmica) de las NTCDS2017, Serie Investigación y Desarrollo SID 706, Instituto de Ingeniería, UNAM.

Ruiz, S E y R Diederich (1989) “The seismic performance of buildings with weak first story”, Earthquake Spectra, Vol. 5, No. 1, pp. 89-102, DOI: 10.1193/1.1585512

Ruiz, S E, M A Santos-Santiago, M A Orellana y R Jiménez (2019), "Fragility analysis of a soft first story building rehabilitated with buckling restrained braces", $12^{\text {th }}$ Canadian Conference on Earthquake Engineering, Quebec, QC, Canadá.

Santos Santiago, M A, S E Ruiz Gómez, y R Jiménez Jordán (2018), “Comparación de tres técnicas de rehabilitación para edificios con planta baja débil”, XV Simposio Nacional de Ingeniería Sísmica, Ciudad de México.

Sues, R H, S T Mau, y Y K Wen (1988), "Systems identification of degrading hysteretic restoring forces", Journal of Engineering Mechanics, Vol. 114, No. 5, pp. 833-846. DOI: 10.1061/(ASCE)0733$\underline{9399(1988) 114: 5(833)}$

Tena-Colunga, A (2010), "Review of the soft first story irregularity condition of buildings for seismic design", The Open Civil Engineering Journal, Vol. 4, pp. 1-15, https://opencivilengineeringjournal.com/contents/volumes/V4/TOCIEJ-4-1/TOCIEJ-4-1.pdf

Terán-Gilmore, A y N Virto-Cambray (2009). "Preliminary design of low-rise buildings stiffened with buckling-restrained braces by a displacement-based approach" Earthquake Spectra, Vol. 25, No. 1, pp. 185 -211. DOI: 10.1193/1.3054638

Terán-Gilmore, A, O Zúñiga-Cuevas y J Ruiz-García (2009). "Displacement-based seismic assessment of low-height confined masonry buildings", Earthquake Spectra, Vol 5, No. 2, pp. 439-464. DOI: $\underline{10.1193 / 1.3111149}$

Vamvatsikos D, y A Cornell (2002), "Incremental dynamic analysis" Earthquake Engineering \& Structural Dynamics Vol. 31, pp. 491-514. DOI: 10.1002/eqe.141 